\title{
From correlation functions to Wilson loops
}

\section{Luis F. Alday, ${ }^{a}$ Burkhard Eden, ${ }^{b}$ Gregory P. Korchemsky, ${ }^{c}$ Juan Maldacena, ${ }^{a}$ and Emery Sokatchev ${ }^{d}$}

${ }^{a}$ School of Natural Sciences, Institute for Advanced Study, Princeton, NJ 08540, U.S.A.

${ }^{b}$ Institut für theoretische Physik, Universität Leipzig, Postfach 100920, D-04009 Leipzig, Germany

${ }^{c}$ Institut de Physique Théorique, ${ }^{1}$ CEA Saclay, 91191 Gif-sur-Yvette Cedex, France

${ }^{d}$ LAPTH, ${ }^{2}$ Université de Savoie, CNRS, B.P. 110, F-74941 Annecy-le-Vieux, France

E-mail: alday@maths.ox.ac.uk, burkhard.eden@durham.ac.uk, gregory.korchemsky@cea.fr, malda@ias.edu, emeri.sokatchev@cern.ch

ABSTRACT: We start with an $n$-point correlation function in a conformal gauge theory. We show that a special limit produces a polygonal Wilson loop with $n$ sides. The limit takes the $n$ points towards the vertices of a null polygonal Wilson loop such that successive distances $x_{i, i+1}^{2} \rightarrow 0$. This produces a fast moving particle that generates a "frame" for the Wilson loop. We explain in detail how the limit is approached, including some subtle effects from the propagation of a fast moving particle in the full interacting theory. We perform perturbative checks by doing explicit computations in $\mathcal{N}=4$ super-Yang-Mills theory.

Keywords: Supersymmetric gauge theory, Extended Supersymmetry, Duality in Gauge Field Theories, AdS-CFT Correspondence

ARXIV EPRINT: 1007.3243

\footnotetext{
${ }^{1}$ Unité de Recherche Associée au CNRS URA 2306.

${ }^{2}$ Laboratoire d'Annecy-le-Vieux de Physique Théorique, UMR 5108.
} 


\section{Contents}

1 Introduction 1

2 From correlators to Wilson loops in dimensionally regularized theories 4

2.1 A short explanation 4

2.2 A more rigorous explanation 5

3 Explicit computation in $\mathcal{N}=4$ super-Yang-Mills $\quad 9$

$\begin{array}{ll}3.1 \text { Computing the correlator with a single insertion } & 11\end{array}$

4 The Wilson loop from the correlation function in four dimensions $\quad 14$

4.1 One-loop checks 23

4.2 A two-loop check for the four point correlator 26

$\begin{array}{llr}5 & \text { Conclusions } & 30\end{array}$

$\begin{array}{ll}\text { A Coordinate changes } & 31\end{array}$

$\begin{array}{ll}\text { B Calculating the integral } I_{\epsilon}(x) & 33\end{array}$

$\begin{array}{ll}\text { C Scalar propagator on the light cone } & 34\end{array}$

D A factor of two $\quad 35$

\section{Introduction}

The natural observables in a conformal field theory are correlation functions of gauge invariant local operators,

$$
G_{n}=\left\langle\mathcal{O}\left(x_{1}\right) \mathcal{O}\left(x_{2}\right) \ldots \mathcal{O}\left(x_{n}\right)\right\rangle .
$$

These correlation functions have well-controlled singularities when two points approach each other, $x_{i} \rightarrow x_{j}$, along a space-like direction. This leads to the well-understood Euclidean operator product expansion (OPE) in terms of local operators. In many applications it is interesting to consider a limit in which the proper distance between two points becomes zero, $x_{i j}^{2} \rightarrow 0$, but the points remain separated along a null direction. This leads to the well studied light-cone OPE which is important for many high energy processes in QCD, such as deep inelastic scattering, etc.

In this paper we consider a situation where the points $x_{i}(i=1, \ldots, n)$ become light-like separated from each other in a sequential fashion, $x_{i, i+1}^{2} \rightarrow 0$ (with the cyclic identification $\left.x_{i+n} \equiv x_{i}\right)$, so that many distances are becoming light-like at the same time. We find that in this limit the leading asymptotic behavior of the correlator $G_{n}$ is given by the 


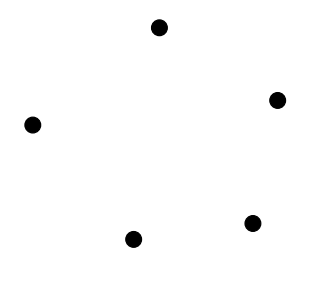

(a)

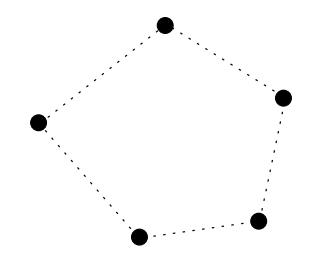

(b)

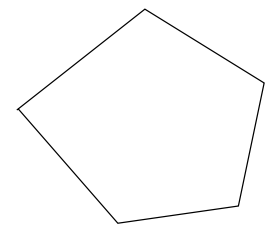

(c)

Figure 1. (a) Diagrammatic representation of the correlation function; the black dots denote the points $x_{i}$. (b) The distances $x_{i, i+1}^{2}$ go to zero. (c) We are left with a Wilson loop on a polygonal contour with null sides.

product of a tree-level correlator and the expectation value of a polygonal Wilson loop operator, defined on a piecewise null polygon $C_{n}$ with cusps at the points $x_{i}$, in the adjoint representation of the gauge group $\mathrm{SU}\left(N_{c}\right)$ :

$$
W^{\text {adj }}\left[C_{n}\right]=\frac{1}{N_{c}^{2}-1}\left\langle 0\left|\operatorname{Tr}_{\text {adj }} \mathrm{P} \exp \left(i g \oint_{C_{n}} d x \cdot A(x)\right)\right| 0\right\rangle .
$$

Taking the limit of the correlator gives (see figure 1)

$$
\lim _{x_{i, i+1}^{2} \rightarrow 0} G_{n} / G_{n}^{\text {tree }} \propto W^{\text {adj }}\left[C_{n}\right],
$$

where the proportionality factor depends on the details of the limit and we will discuss it further below. Here we quoted the result for a theory whose fields are all in the adjoint representation of the gauge group. In the planar limit this can be approximated by a product of a Wilson loop in the fundamental and another one in the anti-fundamental representation of $\mathrm{SU}\left(N_{c}\right)$.

In this way we find a concrete relation between correlation functions of local operators and certain Wilson loop operators. We motivate the connection and provide explicit evidence for the statements we make, in the case of $\mathcal{N}=4$ super-Yang-Mills theory (SYM) in the planar limit. However, the statements regarding the emergence of the Wilson loop should be valid for a general conformal field theory in any space-time dimension.

The basic reason why we obtain the Wilson loop is very simple. When two points are null separated, $x_{i, i+1}^{2} \rightarrow 0$, there is a singularity in the correlator that arises from a very fast particle propagating between these points. In a free theory, this is the ordinary $1 / x_{i, i+1}^{2}$ singularity of the propagator. In the interacting theory, the fast particle going around the various vertices of the polygon is charged under the gauge group, so it interacts with the gauge field. When $x_{i, i+1}^{2} \rightarrow 0$, the propagator of the interacting particle can be approximated by a light-like Wilson line connecting the points $x_{i}$ and $x_{i+1}$. The full approach to the limit is a bit subtle, since one has to carefully regularize the two singular objects in (1.3). Our goal is to explain the limit in detail and to provide several explicit checks of the correctness of the arguments.

The light-cone limit of the correlator can be performed in different ways. The first is to start with $G_{n}$ in four dimensions, with space-like separated points. There the correlator 
is well defined and manifestly conformally covariant. Then one approaches the limit where $x_{i, i+1}^{2} \rightarrow 0$ and the points define a polygonal loop with null sides. In taking this limit one gets leading divergent terms of the type

$$
G_{n} / G_{n}^{\text {tree }} \sim \exp \left(-\frac{\Gamma_{\text {cusp }}^{\text {adj }}}{4} \sum_{i=1}^{n} \log x_{i, i+1}^{2} \log x_{i-1, i}^{2}\right),
$$

where $\Gamma_{\text {cusp }}^{\text {adj }}$ is the cusp anomalous dimension in the adjoint representation of the gauge group. It is known to determine the leading UV singularity of light-like Wilson loops [1-4], ${ }^{1}$ as well as the IR singularity of amplitudes [5-9]. In the present context, the cusp anomalous dimension also gives the leading short-distance singularity of correlators. Thus the distances $x_{i, i+1}^{2}$ serve as a UV regulator for the Wilson loop. In fact, they regulate the Wilson loop in a rather subtle way, since there are further logarithmic terms whose structure depends on more details about the particles propagating along the polygon contour. These details are irrelevant if we only do a one-loop perturbative computation, but they become important when we go to two loops and higher. We elucidate the full structure of the approach to the limit. The final formula is given in (4.18) below.

Another way to take the limit is to first regularize the theory in the ultraviolet by, e.g., formulating it in $D=4-2 \epsilon$ dimensions (with $\epsilon>0$ ). This simplifies the approach to the limit $x_{i, i+1}^{2}=0$. To be more precise, we take the limit so that these distances remain much smaller than the UV regulator scale, $x_{i, i+1}^{2} \ll 1 / \mu^{2}$. This is a well-defined procedure in dimensional regularization. It requires, however, the computation of the correlator in $D=4-2 \epsilon$ dimensions. From a practical point of view, this is harder than the computation in $D=4$ dimensions. From a conceptual point of view, the advantage is that this approach to the limit is much simpler because the theory becomes free in the UV regime. The particle propagators have $1 / x_{i, i+1}^{2}$ poles with no further corrections. Thus, the contribution from these propagators is the same as the one present in the tree-level correlator

$$
\lim _{x_{i, i+1}^{2} \rightarrow 0} \frac{G_{n}}{G_{n}^{\text {tree }}}=W_{\epsilon}^{\text {adj }}\left[C_{n}\right],
$$

where both the left- and right-hand sides have been defined in the dimensionally regulated theory. Of course, this would also be the behavior in any theory that is UV free, such as $(2+1)$-dimensional Yang-Mills theory.

The polygonal Wilson loops (1.2) have been intensively studied during the past few years due to their connection with scattering amplitudes in $\mathcal{N}=4$ super-Yang-Mills [10-20]. The present paper was also motivated by this study. Planar $\mathcal{N}=4$ SYM theory is integrable and the integrability might allow us to compute either Wilson loops or correlation functions. The connection we propose here can be used either as a way to extract a Wilson loop from a known correlator, or as a way to constrain an unknown correlator with the knowledge of the Wilson loop expectation value.

This paper is organized as follows. First we discuss the dimensionally regularized version of the statement (1.5) in section 2. We perform one-loop checks of this statement

\footnotetext{
${ }^{1}$ Our definition of the cusp anomalous dimension differs by a factor of two from the one in [1-4] and other papers in the literature, $\Gamma_{\text {cusp }}^{\text {here }}=2 \Gamma_{\text {cusp }}^{\text {there }}$.
} 
in section 3. In section 4 we take the limit of the full four dimensional correlation function and derive the precise approach to the limit that produces the Wilson loop. Finally, we end with some conclusions. The paper contains several appendices addressing various technical issues.

\section{From correlators to Wilson loops in dimensionally regularized theories}

\subsection{A short explanation}

Let us start with a simple example. Consider the $n$-point correlation function $\left\langle\mathcal{O}\left(x_{1}\right) \cdots \mathcal{O}\left(x_{n}\right)\right\rangle$ of the operators $\mathcal{O}(x)=\operatorname{Tr}\left[\phi^{2}(x)\right]$ in a free theory with $\phi(x)$ being a real scalar field. When we approach the limit $x_{i, i+1}^{2} \rightarrow 0$, the most singular part of the connected correlator goes as

$$
\left\langle\mathcal{O}\left(x_{1}\right) \cdots \mathcal{O}\left(x_{n}\right)\right\rangle \propto \frac{N_{c}^{2}}{\prod_{i=1}^{n} x_{i, i+1}^{2}} .
$$

This is certainly one of the terms contributing to the free correlator. There are other terms which are less singular where some of the contractions involve space-like separated points, see figure 2 .

Let us now consider the same operators in an interacting theory which is dimensionally regularized. We expect that in the limit $x_{i, i+1}^{2} \rightarrow 0$ the leading singularity in the correlator is the same as in the free theory, eq. (2.1). The reason is that we are taking the limit with the regularization scale $\mu^{2}$ kept fixed, $x_{i, i+1}^{2} \mu^{2} \rightarrow 0$, so that when the distances are becoming zero, we are exploring the UV structure of the theory which is free in the regularized theory. Here we are using that dimensional regularization preserves Lorentz invariance, even for distances smaller than the regulator scale.

Naively one would think that the limit is then identical to (2.1). However, the fact that the $\phi$-particles are color charged implies that they source a color electric field. The electric field is not modifying the singular behavior of the correlator, but it leads to a finite contribution

$$
\lim _{x_{i, i+1}^{2} \rightarrow 0} \frac{G_{n}}{G_{n}^{\text {tree }}}=W_{\epsilon}^{\text {adj }}\left[C_{n}\right]=\frac{1}{N_{c}^{2}-1}\left\langle 0\left|\operatorname{Tr}_{\text {adj }} \mathrm{P} \exp \left(i g \oint_{C_{n}} d x \cdot A(x)\right)\right| 0\right\rangle,
$$

where $W_{\epsilon}^{\text {adj }}\left[C_{n}\right]$ is the Wilson loop in the adjoint representation in the dimensionally regularized theory. The contour $C_{n}$ is a polygonal contour where all sides are null and the cusps are located at the limiting positions of the operator insertions. This formula would also be valid in a theory that is UV free such as $(2+1)$-dimensional Yang Mills. ${ }^{2}$

In the planar approximation the adjoint Wilson loop is equal to the product of a Wilson loop in the fundamental and one in the anti-fundamental. In a charge conjugation invariant theory the last two are equal to each other and we can write $W^{\text {adj }}\left[C_{n}\right]=\left(W\left[C_{n}\right]\right)^{2}$.

\footnotetext{
${ }^{2}$ Even though QCD is asymptotically free, the coupling does not decrease fast enough to render these Wilson loops finite in four dimensions.
} 


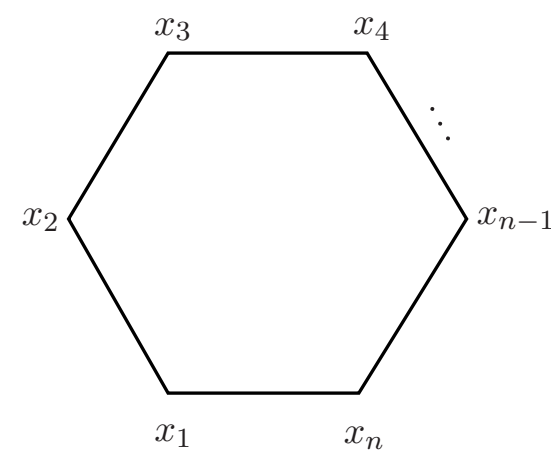

(a)

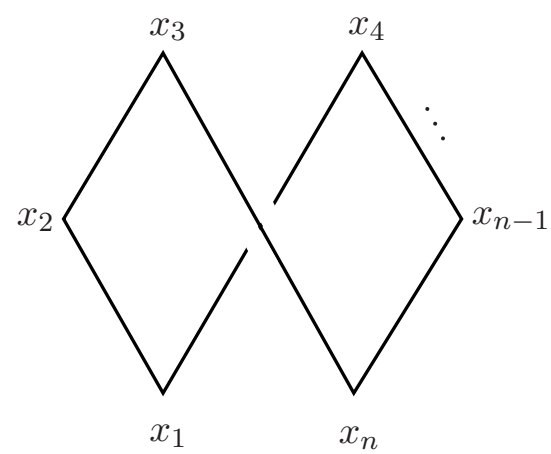

(b)

Figure 2. Feynman diagrams of different types contributing to the correlator (2.1) at tree level. The lines denote free scalar propagators $\left\langle\phi\left(x_{i}\right) \phi\left(x_{j}\right)\right\rangle$. In the light-cone limit $x_{i, i+1}^{2} \rightarrow 0$ the leading contribution comes from diagram (a), while that of diagram (b) is suppressed by the factor $x_{34}^{2} x_{1 n}^{2} /\left(x_{3 n}^{2} x_{14}^{2}\right)$.

\subsection{A more rigorous explanation}

In this section we argue that the correlation functions of protected operators in any conformal field theory have a universal behavior in the light-cone limit $x_{i, i+1}^{2} \rightarrow 0$, similar to that of a polygonal light-like Wilson loop.

At tree level, the $n$-point correlation function $G_{n}^{\text {tree }}$ of operators of the schematic form $\operatorname{Tr}\left[\phi^{2}(x)\right]$ reduces to a sum of products of $n$ free scalar propagators. The corresponding Feynman diagrams can be separated into connected and disconnected ones. The contribution of the connected graphs has the form

$$
G_{n}^{\text {tree }}=N_{c}^{2} \sum_{\left\{i_{1}, \ldots, i_{n}\right\}} S\left(x_{i_{1}, i_{2}}\right) S\left(x_{i_{2}, i_{3}}\right) \ldots S\left(x_{i_{n}, i_{1}}\right)
$$

where $x_{i j}=x_{i}-x_{j}$ and $S(x)=1 /\left(4 \pi^{2} x^{2}\right)$ is the free scalar propagator. Here the sum runs over all non-cyclic permutations of the indices $i_{1}, \ldots, i_{n}$. In the light-cone limit $x_{i, i+1}^{2} \rightarrow 0$ (with $x_{i+n} \equiv x_{i}$ ) the dominant contribution to (2.3) comes from a single term with cyclic ordering of the indices:

$$
G_{n}^{\text {tree }} \stackrel{x_{i, i+1}^{2} \rightarrow 0}{\longrightarrow} N_{c}^{2} S\left(x_{12}\right) S\left(x_{23}\right) \ldots S\left(x_{n 1}\right)=\frac{(2 \pi)^{-2 n} N_{c}^{2}}{x_{12}^{2} x_{23}^{2} \ldots x_{n 1}^{2}} .
$$

Here we have assumed that the operators are such that the contraction leads to a nonvanishing result. If we consider bilinear operators in the $\mathcal{N}=4$ super Yang Mills theory, this statement means that we are choosing Konishi operators or the half-BPS operators in the $\mathbf{2 0}^{\prime}$ in the appropriate fashion. At loop level, we have to take into account that, propagating between the points $x_{i}$ and $x_{i+1}$, the scalar field can interact with gluons, gluinos and with other scalars. Examples of the corresponding diagrams are shown in figure 3. In what follows we shall compute their contribution in two steps. We will first discard the interaction with gluinos and scalars and resum the diagrams shown in figure 3(a) over all possible gluons exchanges. Then, we will take into consideration the remaining interaction 


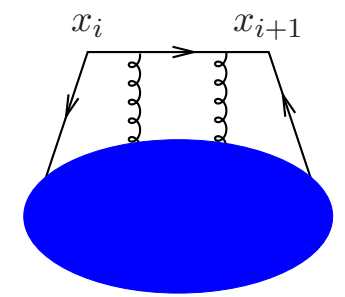

(a)

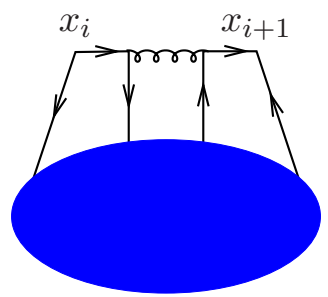

(b)

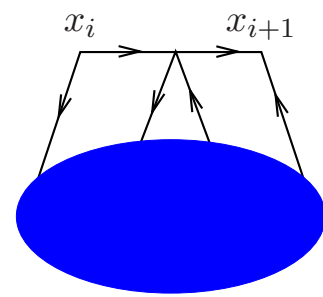

(c)

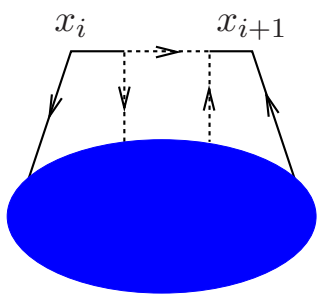

(d)

Figure 3. Different types of diagrams contributing to the correlation function $G_{n}$. Solid, wavy and dashed lines denote scalars, gluons and gluinos, respectively. The vertex with coordinate $x_{i}$ represents the operator $\mathcal{O}\left(x_{i}\right)$. The shadowed blobs stand for the rest of the diagram involving the remaining operators.

vertices shown in figure $3(\mathrm{~b})-(\mathrm{d})$ and argue that their contribution does not affect the leading asymptotic behavior of the correlator for $x_{i, i+1}^{2} \rightarrow 0$.

It is easy to see that the net effect of the interaction of the scalar particle, propagating between the points $x_{i}$ and $x_{i+1}$, with the gauge field amounts to replacing the free scalar propagator, $S\left(x_{i, i+1}\right)$, by the propagator in a background gluon field, $S\left(x_{i}, x_{i+1} ; A\right)$,

$$
G_{n} \stackrel{x_{i, i+1}^{2} \rightarrow 0}{\longrightarrow}\left\langle 0\left|\operatorname{Tr}\left[S\left(x_{1}, x_{2} ; A\right) S\left(x_{2}, x_{3} ; A\right) \ldots S\left(x_{n}, x_{1} ; A\right)\right]\right| 0\right\rangle,
$$

where the expectation value is taken with respect to the $\mathcal{N}=4$ SYM action (3.12). By definition, the scalar propagator in the external field, $S(x, y ; A)$, satisfies the equation

$$
i D^{\mu} D_{\mu} S(x, y ; A)=\delta^{(4-2 \epsilon)}(x-y),
$$

where $D^{\mu}=\partial_{x}^{\mu}-i g\left[A^{\mu}(x),\right]$. Here we have introduced dimensional regularization in order to deal with the short-distances singularities of the correlator for $x_{i, i+1}^{2} \rightarrow 0$.

According to (2.5), the asymptotic behavior of the correlator on the light-cone is related to the behavior of the scalar propagator $S\left(x_{i}, x_{i+1} ; A\right)$ in the limit $x_{i, i+1}^{2} \rightarrow 0$. In this limit, it is convenient to look for a solution to (2.6) of the form

$$
S\left(x_{i}, x_{i+1} ; A\right)=S^{\text {tree }}\left(x_{i, i+1}\right) P \exp \left(i g \int_{x_{i}}^{x_{i+1}} d z \cdot A(z)\right) G\left(x_{i}, x_{i+1} ; A\right),
$$

where we have singled out the free scalar propagator $S^{\text {tree }}\left(x_{i, i+1}\right)=$ $\left(-x_{k, k+1}^{2}\right)^{-1+\epsilon} \Gamma(1-\epsilon) /\left(4 \pi^{2-\epsilon}\right)$ and have factorized the dependence on the gauge field into a path-ordered exponential running along the line connecting the points $x_{i}$ and $x_{i+1}$, and yet another (matrix-valued) function $G\left(x_{i}, x_{i+1} ; A\right)$ to be determined below. Notice that the path-ordered exponential in (2.7) is defined in the adjoint representation of the gauge group, $\left[A_{\mu}(z)\right]^{a b}=i f^{a b c} A_{\mu}^{c}(z)$. It transforms under gauge transformations in the same way as the scalar propagator, $S\left(x_{i}, x_{i+1} ; A^{\Omega}\right)=\Omega^{-1}\left(x_{i}\right) S\left(x_{i}, x_{i+1} ; A\right) \Omega\left(x_{i+1}\right)$. Then the second factor in the right-hand side of (2.7) transforms as follows

$$
G\left(x_{i}, x_{i+1} ; A^{\Omega}\right)=\Omega^{-1}\left(x_{i+1}\right) G\left(x_{i}, x_{i+1} ; A\right) \Omega\left(x_{i+1}\right) .
$$

For our purposes, we need to know the expansion of $G\left(x_{i}, x_{i+1} ; A\right)$ for $x_{i, i+1}^{2} \rightarrow 0$. 
The expansion of the scalar propagator in a background gauge field crucially depends on the hierarchy between the two scales, $1 / x_{i, i+1}^{2}$ and $\mu^{2}$. The former defines the energy carried by the scalar field, while the latter sets up an ultraviolet cutoff on the momentum carried by the gauge field. In this section, we are interested in the limit $x_{i, i+1}^{2} \mu^{2} \ll 1$. It corresponds to the situation where the gluon momentum is much smaller than the energy of the scalar particle. In other words, the propagator (2.7) describes the scattering of an infinitely fast scalar (color charged) particle off a slowly varying background field $A_{\mu}(x)$. Such a scattering only slightly modifies the free scalar propagator by inducing the eikonal phase given by $P \exp \left(i g \int_{x_{i}}^{x_{i+1}} d z \cdot A(z)\right)$. In terms of (2.7) this corresponds to

$$
G\left(x_{i}, x_{i+1} ; A\right) \rightarrow 1, \quad \text { as } x_{i, i+1}^{2} \mu^{2} \rightarrow 0 .
$$

The same result can be derived from the conventional operator product expansion (OPE). Let us return to (2.7) and examine the asymptotic behavior of the scalar propagator at short distances, $x_{i, i+1}^{2} \rightarrow 0$. It is convenient to choose $x_{i}=x, x_{i+1}=0$ and study $G(x, 0 ; A)$ for $x^{2} \rightarrow 0$. In this limit, we can apply the OPE and expand $G(x, 0 ; A)$ over an (infinite) set of local operators. Then, taking into account (2.8), we observe that the expansion of $G(x, 0 ; A)$ at small $x^{2}$ should run over local operators $\mathcal{O}^{\mu_{1} \ldots \mu_{N}}(0)$ built from the gauge field strength $F_{\mu \nu}$ and the covariant derivatives $D_{\mu}$ at the origin,

$$
G(x, 0 ; A)=\sum_{N, \Delta}\left(x^{2}\right)^{\Delta} C_{\Delta, N}\left(x^{2} \mu^{2}\right) x_{\mu_{1}} \ldots x_{\mu_{N}} \mathcal{O}_{\Delta}^{\mu_{1} \ldots \mu_{N}}(0),
$$

where the expansion goes over local operators carrying Lorentz spin $N$ and canonical dimension $\ell_{\mathcal{O}}$. Here $C_{\Delta, N}\left(x^{2} \mu^{2}\right)$ are dimensionless coefficient functions and the Wilson operators $\mathcal{O}^{\mu_{1} \ldots \mu_{N}}(0)$ are normalized at the scale $\mu^{2}$. For $x^{2} \rightarrow 0$ the contribution of local operators to the right-hand side of (2.10) is suppressed as $\left(x^{2}\right)^{\Delta}$. It follows from the dimensional counting that the exponent $\Delta$ is determined by the twist of the operator $\tau=\ell_{\mathcal{O}}-N$

$$
2 \Delta+N=\ell_{\mathcal{O}} \quad \rightarrow \quad \Delta=\tau / 2,
$$

with $\tau$ taking non-negative integer values. If the twist of $\mathcal{O}_{\Delta}^{\mu_{1} \ldots \mu_{N}}$ is non-vanishing, $\tau=$ $1,2, \ldots$, we have $\Delta>0$; if instead $\tau=0$, which corresponds to the identity operator, we find $\Delta=0$. So, the leading contribution to (2.10) in the limit $x^{2} \rightarrow 0$ comes from the identity operator. ${ }^{3}$

Notice that the relation (2.10) holds for arbitrary $x^{2}$ and $\mu^{2}$. The physical meaning of (2.10) is that the OPE separates the contributions from large and short distances, compared to $1 / \mu^{2}$, into local operators and coefficient functions, respectively. Let us examine (2.10) in two different limits: $x^{2} \mu^{2} \ll 1$ and $x^{2} \mu^{2} \gg 1$. In dimensional regularization, the perturbative expansion of the coefficient functions $C_{\Delta, N}\left(x^{2} \mu^{2}\right)$ goes in powers of $g^{2}\left(x^{2} \mu^{2}\right)^{\epsilon}$. As a consequence, for $x^{2} \mu^{2} \rightarrow 0$ the loop corrections to the coefficient function $C_{\Delta, N}\left(x^{2} \mu^{2}\right)$ vanish order by order in the coupling constant and we recover the expected

\footnotetext{
${ }^{3}$ The explicit expression for the subleading contribution of the twist-two operators to (2.10) can be found in appendix $\mathrm{C}$.
} 
result (2.9). At the same time, for $x^{2} \mu^{2} \gg 1$ the coefficient function is different from unity and needs to be taken into account.

So far we considered the contribution of the diagrams shown in figure 3(a). Let us now examine the remaining diagrams in figures $3(\mathrm{~b})-(\mathrm{d})$. In a close analogy with the previous case, we can interpret them as contributing to the propagator of a scalar particle in the background of the other scalars and gluinos. As before, the short-distance behavior of the propagator can be studied using the OPE (2.10). The net effect of the diagrams in figures 3(b)(d) is to enlarge the set of local operators contributing to the right-hand side of (2.10). Namely, the resulting local operators will involve additional pairs of scalars and gluinos. Most importantly, the twist of such operators is greater than two. Consequently, their contribution to (2.10) is suppressed as $x^{2} \rightarrow 0$ and, therefore, it can be safely discarded.

Finally, we combine together the relations (2.7) and (2.9) and obtain the leading asymptotic behavior of the propagator on the light cone $x_{i, i+1}^{2} \mu^{2} \rightarrow 0$ as [7-9, 21]

$$
S\left(x_{i}, x_{i+1} ; A\right) \rightarrow S^{\text {tree }}\left(x_{i, i+1}\right) P \exp \left(i g \int_{x_{i}}^{x_{i+1}} d z \cdot A(z)\right) .
$$

This relation has a transparent physical interpretation. In the first-quantized picture, the propagator $S\left(x_{i}, x_{i+1} ; A\right)$ describes the transition amplitude for a charged massless particle to go from point $x_{i}$ to $x_{i+1}$. As such, it is given by the sum over all paths $C_{x_{i}, x_{i+1}}$ connecting these two points. The interaction of the particle with the external gauge field brings in an additional weight factor given by the path-ordered exponential evaluated along $C_{x_{i}, x_{i+1}}$. In the limit $x_{i, i+1}^{2} \mu^{2} \rightarrow 0$, corresponding to the propagation of an infinitely fast particle along the light cone, the path integral is dominated by the saddle point contribution. The latter is just the classical trajectory of the particle, that is, the straight line connecting points $x_{i}$ and $x_{i+1}$.

Let us now replace the scalar propagators in (2.5) by their leading asymptotic behavior $(2.12)$. We find that the product of free propagators reproduces the correlator at tree level, eq. (2.4), whereas the path-ordered exponentials are combined into a single factor,

$$
G_{n} \rightarrow G_{n}^{\text {tree }} W^{\text {adj }}\left[C_{n}\right]
$$

where $G_{n}^{\text {tree }}$ is the tree-level correlator and $W^{\text {adj }}\left[C_{n}\right]$ is a Wilson loop in the adjoint representation, evaluated along the light-like polygon $C_{n}=\left[x_{1}, x_{2}\right] \cup\left[x_{2}, x_{3}\right] \cup \ldots \cup\left[x_{n}, x_{1}\right]$ :

$$
W^{\text {adj }}\left[C_{n}\right]=\frac{1}{N_{c}^{2}-1}\left\langle 0\left|\operatorname{Tr}_{\text {adj }}\left[P \exp \left(i g \int_{C_{n}} d z \cdot A(z)\right)\right]\right| 0\right\rangle .
$$

Here the overall normalization factor $1 /\left(N_{c}^{2}-1\right)$ is inserted in order for $W^{\text {adj }}\left[C_{n}\right]$ to be 1 at the lowest order in the coupling constant. Since the tree-level expression $G_{n}^{\text {tree }}$ is not well defined for $x_{i, i+1}^{2}=0$, we can rewrite $(2.13)$ in the form

$$
\lim _{x_{i, i+1}^{2} \rightarrow 0}\left(\frac{G_{n}}{G_{n}^{\text {tree }}}\right)=W^{\text {adj }}\left[C_{n}\right] .
$$

In section 3 we check the validity of this relation by an explicit one-loop calculation in $\mathcal{N}=4$ SYM theory. 
Notice that the Wilson loop $W^{\text {adj }}\left[C_{n}\right]$ contains UV divergences due to the cusps on the contour. This does not contradict, however, the UV finiteness of the correlator of protected operators $G_{n}$. It is only in the light-cone limit, $x_{i, i+1}^{2} \rightarrow 0$, that the correlator develops new, light-cone singularities. According to (2.15), they are in one-to-one correspondence with the ultraviolet divergences of the light-like Wilson loop.

We wish to emphasize that the Wilson loop in the right-hand side of $(2.15)$ is defined in the adjoint representation of the color group $\mathrm{SU}\left(N_{c}\right)$. This has to do with the fact that the $\mathcal{N}=4 \mathrm{SYM}$ scalars belong to this representation. The relation (2.15) can be further simplified in the multi-color limit by using the well-known property of the Wilson loops mentioned at the end of section 2.1,

$$
W^{\operatorname{adj}}\left[C_{n}\right]=\left(W\left[C_{n}\right]\right)^{2}+O\left(1 / N_{c}^{2}\right),
$$

where $W\left[C_{n}\right]$ is defined in the fundamental representation of $\mathrm{SU}\left(N_{c}\right)$, see eq. (3.9). The relation (2.16) becomes very useful when comparing correlators with planar gluon MHV scattering amplitudes, since the latter are related to Wilson loops $W\left[C_{n}\right]$ in the fundamental representation.

\section{Explicit computation in $\mathcal{N}=4$ super-Yang-Mills}

In this section we perform some explicit computations in $\mathcal{N}=4$ super-Yang-Mills to confirm the above picture. The reader who is interested only in general statements can jump to the next section.

In the $\mathcal{N}=4 \mathrm{SYM}$ theory there are two types of gauge invariant operators, protected and non-protected. The former are not renormalized and thus have a fixed conformal dimension, equal to their canonical dimension. The simplest example is the bilinear gauge invariant operator made of the six real scalars in the vector multiplet, $\phi_{A B}=-\phi_{B A}=$ $\frac{1}{2} \epsilon_{A B C D} \bar{\phi}^{C D}$, where $A, B=1,2,3,4$ are indices of the fundamental irrep of the R symmetry group $\mathrm{SU}(4)$. The bilinear

$$
\mathcal{O}_{A B C D}=\operatorname{Tr}\left(\phi_{A B} \phi_{C D}\right)-\frac{1}{12} \epsilon_{A B C D} \operatorname{Tr}\left(\bar{\phi}^{E F} \phi_{E F}\right)
$$

belongs to the irrep $\mathbf{2 0}^{\prime}$ of $\mathrm{SU}(4)$. This scalar operator is the superconformal primary state of a whole tower of protected operators containing, among others, the stress tensor of the theory. Such "short", or half-BPS operators (also known as CPO) have been extensively studied (see, e.g., [22]) in the context of the AdS/CFT correspondence [23-25]. The best known example of an unprotected (i.e., having an anomalous dimension) operator is the so-called Konishi operator

$$
\mathcal{K}=\operatorname{Tr}\left(\bar{\phi}^{A B} \phi_{A B}\right) .
$$

Here we present two examples which illustrate how the correlator becomes a Wilson loop in the light-cone limit. The first is a correlator involving only protected operators, in the second we replace some of these by Konishi operators. In the first case, it is sufficient to consider only certain projections of $\mathbf{2 0}^{\prime}$, namely

$$
\mathcal{O}=\operatorname{Tr}\left(\phi_{12} \phi_{12}\right), \quad \tilde{\mathcal{O}}=\operatorname{Tr}\left(\bar{\phi}^{12} \bar{\phi}^{12}\right)
$$


where $\mathcal{O}$ is the highest-weight state and $\tilde{\mathcal{O}}$ is the conjugate lowest-weight state. We want to evaluate the correlator of $n=2 m$ operators of the form ${ }^{4}$

$$
G_{n}=\left\langle\mathcal{O}\left(x_{1}\right) \tilde{\mathcal{O}}\left(x_{2}\right) \ldots \mathcal{O}\left(x_{n-1}\right) \tilde{\mathcal{O}}\left(x_{n}\right)\right\rangle .
$$

Such correlators are finite and conformally covariant, because the operators $\mathcal{O}$ are not renormalized. Moreover, it is known that the two- and three-point correlators are themselves protected, i.e. they do not receive quantum corrections beyond tree level [26-30]. Here we are interested in the loop corrections to $G_{n}$, therefore we consider the cases $n \geq 4$. Then, the loop corrections to $G_{n}$ are given by conformally invariant space-time integrals. ${ }^{5}$

Next, we wish to take the limit when the neighboring points become light-like separated,

$$
x_{i, i+1}^{2} \rightarrow 0, \quad i=1, \ldots, n
$$

(assuming that $x_{n+1} \equiv x_{1}$ ). This limit is singular for two reasons. Firstly, the correlator develops pole singularities, as can be seen already from the (connected, planar) tree-level approximation

$$
G_{n}^{\text {tree }}=\frac{(2 \pi)^{-2 n} N_{c}^{2}}{x_{12}^{2} x_{23}^{2} \ldots x_{n 1}^{2}}+\text { subleading terms } .
$$

By "subleading" we mean terms corresponding to different Wick contractions of the fields $\phi$ which are less singular in the limit (3.5) (see an illustration in figure 2). This can be remedied by considering the ratio

$$
\lim _{x_{i, i+1}^{2} \rightarrow 0} G_{n} / G_{n}^{\text {tree }} .
$$

Secondly, the loop integrals develop short-distance (ultraviolet) logarithmic divergences, which can be regularized by computing the correlator in $D=4-2 \epsilon$ (with $\epsilon>0$ ) dimensions. Our aim in this section is to show the relation

$$
\lim _{x_{i, i+1}^{2} \rightarrow 0} G_{n} / G_{n}^{\text {tree }}=\left(W\left[C_{n}\right]\right)^{2},
$$

where $W\left[C_{n}\right]$ is the expectation value of a Wilson loop evaluated on a polygonal light-like contour $C_{n}$ with $n$ cusps at the points $x_{i}$,

$$
W\left[C_{n}\right]=\frac{1}{N_{c}}\left\langle 0\left|\operatorname{tr} \mathrm{P} \exp \left(i g \oint_{C_{n}} d x \cdot A(x)\right)\right| 0\right\rangle,
$$

and computed in the same UV regularization scheme as the correlator. Here $W\left[C_{n}\right]$ is defined in the fundamental representation of $\mathrm{SU}\left(N_{c}\right)$, with $A_{\mu}(x)=A_{\mu}^{a} t^{a}$ and $t^{a}$ being the generators of the fundamental representation. To lowest order in the coupling, the Wilson loop takes the form

$$
W\left[C_{n}\right]=1+\frac{1}{4}(i g)^{2} N_{c} \oint_{C_{n}} d x^{\mu} \oint_{C_{n}} d y^{\nu} D_{\mu \nu}(x-y)+O\left(g^{4}\right),
$$

where $D_{\mu \nu}(x)$ is the free gluon propagator.

\footnotetext{
${ }^{4}$ For $n=2 m+1$ we can add, e.g., one real operator $\hat{\mathcal{O}}=\operatorname{Tr}\left(\bar{\phi}^{12} \phi_{12}\right)-\frac{1}{12} \operatorname{Tr}\left(\bar{\phi}^{E F} \phi_{E F}\right)$.

${ }^{5}$ For an extensive study of such correlators in the case $n=4$, at one and two loops, see, e.g., [31-33] and references therein.
} 
The expression in the right-hand side of (3.8) can be rewritten as a Wilson loop $W^{\text {adj }}\left[C_{n}\right]$ in the adjoint representation of $\mathrm{SU}\left(N_{c}\right)$, see eqs. (2.14) and (2.16). The reason why we prefer to formulate the relation (3.8) in terms of the Wilson loops $W\left[C_{n}\right]$ is that they are known to match planar gluon MHV scattering amplitudes [10-12].

The correlator (3.4) is given by the path integral

$$
G_{n}=\int \mathcal{D} \Phi e^{i \int d^{4} x L(x)} \mathcal{O}\left(x_{1}\right) \ldots \tilde{\mathcal{O}}\left(x_{n}\right),
$$

where $\Phi$ denotes all the fields of the theory. The Lagrangian of $\mathcal{N}=4 \mathrm{SYM}$ has the form

$$
\begin{aligned}
L_{\mathcal{N}=4}= & \operatorname{Tr}\left\{\frac{1}{2} F_{\mu \nu} F^{\mu \nu}+\frac{1}{2}\left(D_{\mu} \bar{\phi}^{A B}\right)\left(D^{\mu} \phi_{A B}\right)+\frac{1}{8} g^{2}\left[\bar{\phi}^{A B}, \bar{\phi}^{C D}\right]\left[\phi_{A B}, \phi_{C D}\right]\right. \\
& \left.+2 i \bar{\lambda}_{\dot{\alpha} A} \sigma_{\mu}^{\dot{\alpha} \beta} D^{\mu} \lambda_{\beta}^{A}-\sqrt{2} g \lambda^{\alpha A}\left[\phi_{A B}, \lambda_{\alpha}^{B}\right]+\sqrt{2} g \bar{\lambda}_{\dot{\alpha} A}\left[\bar{\phi}^{A B}, \bar{\lambda}_{B}^{\dot{\alpha}}\right]\right\}
\end{aligned}
$$

with $D^{\mu}=\partial^{\mu}-i g\left[A^{\mu},\right]$.

Instead of computing the loop corrections to this correlator directly, we prefer to use the well-known procedure of differentiating $G_{n}$ with respect to the coupling $g$. Before doing this, we rescale the gauge field,

$$
A_{\mu} \rightarrow g^{-1} A_{\mu}
$$

As a result, the gluon Lagrangian picks an overall factor, $\left(1 / 2 g^{2}\right) \operatorname{Tr}\left(F_{\mu \nu} F^{\mu \nu}\right)^{2}$, and the coupling drops out of the covariant derivatives, $D^{\mu}=\partial^{\mu}-i\left[A^{\mu},\right]$. Then the derivative of $G_{n}$ with respect to the coupling produces

$$
\begin{aligned}
g^{2} \frac{\partial}{\partial g^{2}} G_{n}\left(x_{1}, \ldots, x_{n}\right) & =-i \int \mathcal{D} \Phi e^{i \int d^{4} x L(x)} \int d^{4} x_{0} L^{\prime}\left(x_{0}\right) \mathcal{O}\left(x_{1}\right) \ldots \tilde{\mathcal{O}}\left(x_{n}\right) \\
& \equiv-i \int d^{4} x_{0} \mathcal{G}_{n+1}\left(x_{0}, x_{1}, \ldots, x_{n}\right)
\end{aligned}
$$

Here

$$
\mathcal{G}_{n+1}=\left\langle L^{\prime}\left(x_{0}\right) \mathcal{O}\left(x_{1}\right) \ldots \tilde{\mathcal{O}}\left(x_{n}\right)\right\rangle
$$

is a new, $(n+1)$-point correlator obtained by inserting the derivative of the Lagrangian

$$
L^{\prime}=\operatorname{Tr}\left\{-\frac{1}{2 g^{2}} F_{\mu \nu} F^{\mu \nu}+\frac{g^{2}}{8}\left[\bar{\phi}^{A B}, \bar{\phi}^{C D}\right]\left[\phi_{A B}, \phi_{C D}\right]-\frac{g}{\sqrt{2}} \lambda^{\alpha A}\left[\phi_{A B}, \lambda_{\alpha}^{B}\right]+\frac{g}{\sqrt{2}} \bar{\lambda}_{\dot{\alpha} A}\left[\bar{\phi}^{A B}, \bar{\lambda}_{B}^{\dot{\alpha}}\right]\right\}
$$

into the original correlator. This new correlator is then integrated in (3.14) over the insertion point $x_{0}$, which generates the loop corrections to $G_{n}$. The advantage of this procedure is that we gain one perturbative order. In particular, the one-loop correction $G_{n}^{(1)}$ is determined by the tree- (or Born) level correlator $\mathcal{G}_{n+1}^{(0)}$.

\subsection{Computing the correlator with a single insertion}

We need to evaluate $\mathcal{G}_{n+1}$ at tree level. The rescaling of the gauge field (3.13) modifies the gluon propagator by a factor of $g^{2}$, while the vertices involving the gauge field now appear without the coupling. In addition, the various terms in the Lagrangian insertion 


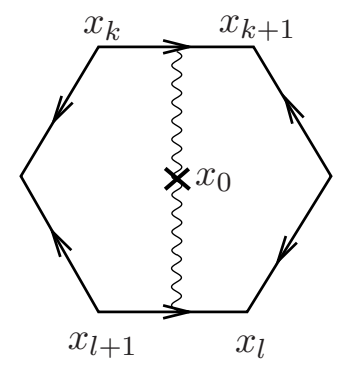

(a)

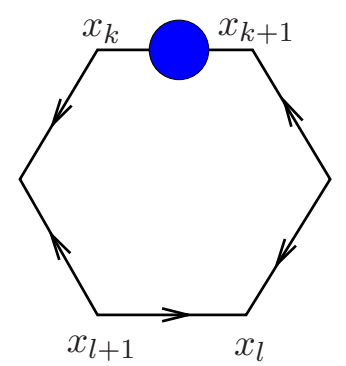

(b)

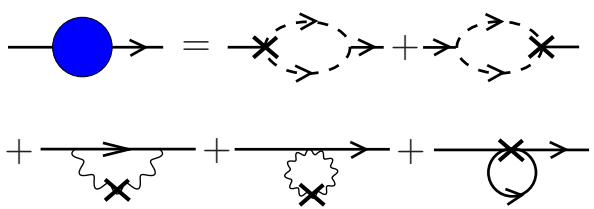

(c)

Figure 4. The leading contribution to the correlator (3.15) in the light-cone limit. Solid, wavy and dashed lines denote complex scalars, gluons and gluinos, correspondingly. The cross denotes the insertion of the derivative of the Lagrangian with respect to the coupling constant $L^{\prime}\left(x_{0}\right)$, eq. (3.16). The big blob in diagram (b) denotes the corrections to the scalar propagator shown in (c).

in (3.15) have their own factors of $g$. Putting all these factors together, we confirm that the tree-level correlator $\mathcal{G}_{n+1}^{(0)}$ is of order $g^{2}$, in accordance with (3.14). Inspecting the terms in the inserted Lagrangian $L^{\prime}$, we see that there exist two types of Feynman graphs at this order shown in figure $4 .{ }^{6}$

After integration over the insertion point, the graphs in figure 4(b), (c) give the correction to the two-point function (propagator) $\left\langle\bar{\phi}\left(x_{k}\right) \phi\left(x_{k+1}\right)\right\rangle$. As explained in section 2.2, we are interested in the particular limit $x_{i, i+1}^{2} \rightarrow 0$, by keeping $\mu^{2} x_{i, i+1}^{2} \ll 1$. In this limit, on dimensional grounds,

$$
\lim _{x_{k, k+1}^{2} \rightarrow 0} \frac{\left\langle\bar{\phi}\left(x_{k}\right) \phi\left(x_{k+1}\right)\right\rangle}{\left\langle\bar{\phi}\left(x_{k}\right) \phi\left(x_{k+1}\right)\right\rangle^{\text {tree }}}=\lim _{\mu^{2} x_{k, k+1}^{2} \rightarrow 0}\left[1+g^{2}\left(\mu^{2} x_{k, k+1}^{2}\right)^{\epsilon} C(\epsilon)+O\left(g^{4}\right)\right]=1 .
$$

So, these graphs do not contribute on the light cone.

The non-trivial contribution comes from the graph in figure 4(a). It contains the gauge kinetic term $F^{2}$ at the insertion point. ${ }^{7}$ Summing up all such graphs, we get the following ratio

$$
G_{n} / G_{n}^{\text {tree }}=-i g^{2} N_{c} \sum_{k \neq l=1}^{n} \frac{\int d^{D} x_{0} T^{\mu \nu}\left(x_{k}, x_{0}, x_{k+1}\right) T_{\mu \nu}\left(x_{l}, x_{0}, x_{l+1}\right)}{S\left(x_{k, k+1}\right) S\left(x_{l, l+1}\right)}+O\left(g^{4}\right),
$$

where $S(x)$ is the free scalar propagator in $D=4-2 \epsilon$ dimensions,

$$
\left\langle\bar{\phi}_{a}\left(x_{k}\right) \phi_{b}\left(x_{k+1}\right)\right\rangle^{\text {tree }}=\delta_{a b} S\left(x_{k, k+1}\right)=\delta_{a b} \frac{\Gamma(1-\epsilon)}{4 \pi^{2-\epsilon}}\left(-x_{k, k+1}^{2}+i 0\right)^{-1+\epsilon},
$$

and $T^{\mu \nu}\left(x_{k}, x_{0}, x_{k+1}\right)$ is defined by the three-point correlator

$$
\left\langle\bar{\phi}_{a}\left(x_{k}\right) F_{b}^{\mu \nu}\left(x_{0}\right) \phi_{c}\left(x_{k+1}\right)\right\rangle=g f_{a b c} T^{\mu \nu}\left(x_{k}, x_{0}, x_{k+1}\right) .
$$

\footnotetext{
${ }^{6}$ Notice that the graphs are drawn with a polygonal matter frame. Graphs based on the "zigzag" configurations like in figure 2(b) are suppressed in the light-cone limit (3.5), after dividing out the leading singularity of the tree-level correlator (3.6).

${ }^{7}$ The quartic scalar term $g^{2} \operatorname{Tr}\left(\left[\bar{\phi}^{A B}, \bar{\phi}^{C D}\right]\left[\phi_{A B}, \phi_{C D}\right]\right)$ from (3.16) does not appear due to the special choice of the external scalars.
} 
To lowest order in the coupling it has the form (we use Feynman gauge for the gluon propagator)

$$
\begin{aligned}
T^{\mu \nu}\left(x_{k}, x_{0}, x_{k+1}\right) & =\int d^{4-2 \epsilon} x_{0^{\prime}}\left(S\left(x_{k, 0^{\prime}}\right) \stackrel{\leftrightarrow}{\partial}_{x_{0^{\prime}}}^{\lambda} S\left(x_{k+1,0^{\prime}}\right)\right)\left(\delta_{\lambda}^{[\mu} \partial_{x_{0}}^{\nu]} S\left(x_{00^{\prime}}\right)\right) \\
& =\kappa_{\epsilon}^{3} \partial_{k}^{[\mu} \partial_{k+1}^{\nu]} \int \frac{d^{4-2 \epsilon} x_{0^{\prime}}}{\left(-x_{k, 0^{\prime}}^{2} x_{k+1,0^{\prime}}^{2} x_{00^{\prime}}^{2}\right)^{1-\epsilon}} \equiv \kappa_{\epsilon}^{3} x_{k, 0}^{[\mu} x_{k+1,0}^{\nu]} I_{\epsilon}\left(x_{k 0}, x_{k+1,0}\right),
\end{aligned}
$$

where $\kappa_{\epsilon}=\Gamma(1-\epsilon) /\left(4 \pi^{2-\epsilon}\right)$ and $\stackrel{\leftrightarrow}{\partial}_{x}=\frac{1}{2}\left(\vec{\partial}_{x}-\overleftarrow{\partial}_{x}\right)$

If we keep the points $x_{k}$ and $x_{k+1}$ separated, $x_{k, k+1}^{2} \neq 0$, the integral in (3.21) converges and we can set $D=4$, i.e., $\epsilon=0$. In this case the scalar integral $I_{0}$ becomes conformally covariant and can easily be evaluated (see, e.g., [34, 35] and also appendix B),

$$
I_{\epsilon=0}\left(x_{k 0}, x_{k+1,0}\right)=-\frac{4 i \pi^{2}}{x_{k, k+1}^{2} x_{k, 0}^{2} x_{k+1,0}^{2}} .
$$

In the limit $x_{k, k+1}^{2} \rightarrow 0$ the integral diverges and we have to stay in $D=4-2 \epsilon$ dimensions. In appendix B we show that for $x_{k, k+1}^{2} \rightarrow 0$

$$
I_{\epsilon}\left(x_{k 0}, x_{k+1,0}\right) \rightarrow-i(1-\epsilon) \kappa_{\epsilon}^{-1}\left(-x_{k, k+1}^{2}\right)^{-1+\epsilon} \int_{0}^{1} d s\left[-\left(x_{k, 0} s+x_{k+1,0} \bar{s}\right)^{2}\right]^{-2+\epsilon},
$$

with $\bar{s}=1-s$. We see that the asymptotic singular behavior of the vertex correction (3.21) for $x_{k, k+1}^{2} \rightarrow 0$ is the same as that of the free propagator (3.19).

Next, replacing $I_{\epsilon}(x)$ in (3.21) by its asymptotic form (3.23) and substituting the vertex corrections in (3.18) yields

$$
\lim _{x_{i, i+1}^{2} \rightarrow 0} G_{n} / G_{n}^{\text {tree }}=-2 i g^{2} N_{c}\left[(1-\epsilon) \kappa_{\epsilon}\right]^{2} \sum_{k \neq l=1}^{n} \int_{0}^{1} d s_{k} d s_{l} \int d^{4-2 \epsilon} x_{0} \frac{x_{a 0}^{[\mu} x_{k, k+1}^{\nu]}\left(x_{b 0}\right)_{\mu}\left(x_{l, l+1}\right)_{\nu}}{\left[x_{a 0}^{2} x_{b 0}^{2}\right]^{2-\epsilon}},
$$

with the notation

$$
x_{a}=x_{k} s_{k}+x_{k+1} \bar{s}_{k}, \quad x_{b}=x_{l} s_{l}+x_{l+1} \bar{s}_{l} .
$$

Up to a normalization factor the above integral can be rewritten as

$$
-\frac{i}{2} \kappa_{\epsilon}^{2} x_{k, k+1}^{\mu} x_{l, l+1}^{\nu}\left[\left(\partial_{a}\right)_{\mu}\left(\partial_{a}\right)_{\nu}-g_{\mu \nu} \square_{a}\right] \int \frac{d^{4-2 \epsilon} x_{0}}{\left[x_{0 a}^{2} x_{0 b}^{2}\right]^{1-\epsilon}}=-\frac{1}{2} x_{k, k+1}^{\mu} x_{l, l+1}^{\nu} D_{\mu \nu}\left(x_{a b}\right),
$$

where $D_{\mu \nu}\left(x_{a b}\right)$ is the gluon propagator in the Landau gauge

$$
D_{\mu \nu}(x)=-i \int \frac{d^{4-2 \epsilon} k}{k^{2}} \mathrm{e}^{i k x}\left(g^{\mu \nu}-\frac{k^{\mu} k^{\nu}}{k^{2}}\right) .
$$

Returning to (3.24), we conclude that

$$
\begin{aligned}
\lim _{x_{i, i+1}^{2} \rightarrow 0} G_{n} / G_{n}^{\text {tree }} & =\frac{1}{2}(i g)^{2} N_{c} \sum_{k \neq l} \int_{0}^{1} d s_{k} \int_{0}^{1} d s_{l} x_{k, k+1}^{\mu} x_{l, l+1}^{\nu} D_{\mu \nu}\left(x_{a b}\right)+O\left(g^{4}\right) \\
& =2 \log W\left[C_{n}\right]
\end{aligned}
$$


which is just the one-loop expression for the light-like Wilson loop (3.10) in the fundamental representation of the $\mathrm{SU}\left(N_{c}\right)$ gauge group, calculated in the Landau gauge. Since the Wilson loop is gauge invariant, we can claim that this result is equivalent to the earlier Wilson loop calculations in the Feynman gauge $[11,12]$. The latter were shown to reproduce the $n$-gluon one-loop amplitude. ${ }^{8,9}$

Finally, let us discuss the case of the one-loop correlator

$$
\langle\mathcal{K}(1) \mathcal{K}(2) O(3) \tilde{O}(4)\rangle,
$$

involving two copies of the Konishi operator (3.2), in addition to two other protected ones. The graphs we have listed before all occur in the same way in this calculation, and in addition we find a contribution from the insertion of the scalar $\phi^{4}$-potential. Divided by the tree, the new terms in the connected part are, up to an overall factor ${ }^{10}$

$$
\begin{aligned}
& x_{12}^{2} x_{14}^{2}\left(g(1,1,2,4)+\frac{x_{23}^{2}}{x_{24}^{2}} g(1,1,2,3)+\frac{x_{34}^{2}}{x_{24}^{2}} g(1,1,3,4)\right) \\
& +x_{12}^{2} x_{23}^{2}\left(g(1,2,2,3)+\frac{x_{14}^{2}}{x_{13}^{2}} g(1,2,2,4)+\frac{x_{34}^{2}}{x_{13}^{2}} g(2,2,3,4)\right) \\
& +2 x_{14}^{2} x_{23}^{2} g(1,2,3,4) \rightarrow 0, \quad \text { when } \quad x_{i, i+1}^{2} \rightarrow 0 .
\end{aligned}
$$

In figure 5 we have depicted the graphs from the first and the last line. It is important here to work with the bare operators, and to use the stronger form of the light-cone limit, in which all $x_{i, i+1}^{2}=\delta$ go to zero at the same rate. We conclude that the correlator (3.29) involving unprotected operators also becomes a Wilson loop in the light-cone limit.

\section{The Wilson loop from the correlation function in four dimensions}

In this section we examine the behavior of the four-dimensional correlation function (1.1) as we take the combined light-like limit $x_{i, i+1}^{2} \rightarrow 0$. We start from the full renormalized four dimensional correlation function defined at space-like separated points and then we take these points to approach the vertices of a polygonal null Wilson loop, making all distances of the form $x_{i, i+1}^{2}$ light-like. In the language of the previous section, we first remove the regulator and then we take $x_{i, i+1}^{2}$ to zero. We will consider first a weakly coupled theory and operators of dimension two which are constructed from two fields in the free theory.

\footnotetext{
${ }^{8}$ The fact that the integrals in the one-loop Wilson loop can be rewritten in a form equivalent to the two-mass easy box integrals, after a suitable identification of the regularization parameters, was already pointed out in [36].

${ }^{9}$ We remark that the effective gauge change, from Feynman to Landau, observed above is not surprising. Indeed, as discussed in [31, 32], inserting the gauge-invariant Lagrangian into a gluon propagator in any gauge brings this propagator to the transverse Landau gauge. So, what happens, at least at one loop, is that if we compute the correlator in dimensional regularization by inserting the Lagrangian, we obtain a result identical with the Wilson loop calculation done in the Landau gauge. This is in contrast with the situation where one calculates the one-loop correlator in the Feynman gauge, but without insertions. There the correlator is expressed in terms of more complicated integrals (see [34, 35]), which coincide with the one-loop scalar box in $D=4$ (off the light cone), but require a special investigation in $D=4-2 \epsilon$ (on the light cone).

${ }^{10}$ The one-loop scalar integral $g(1,2,3,4)$ is taken from $(4.20)$, with a $(4-2 \epsilon)$-dimensional measure.
} 

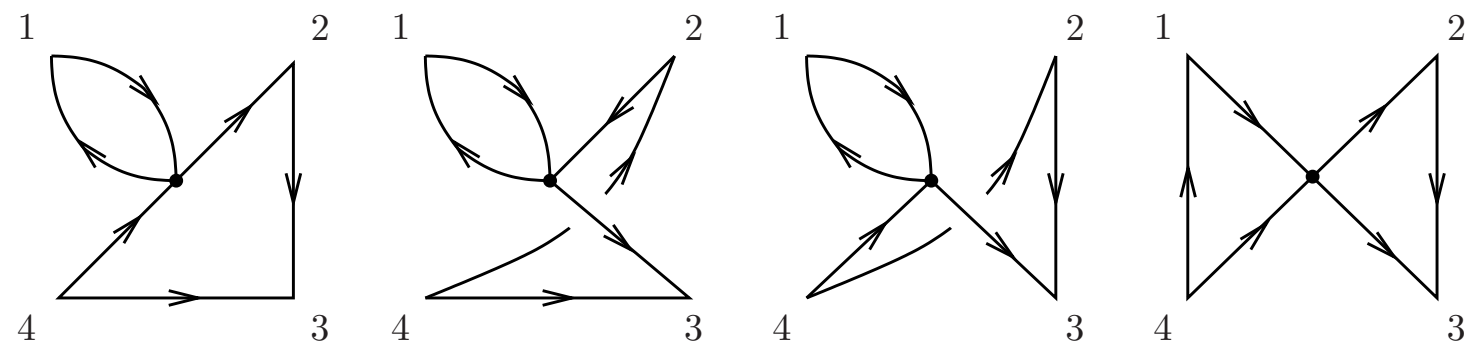

Figure 5. Additional graphs with a $\phi^{4}$-coupling contributing to the correlation function (3.29). The contribution of the first three diagrams is given by the first line in (3.30) while the contribution of the forth diagram is given by the last line in (3.30).

As we take the light-like limit the correlator develops some singularities. Thus we need to understand precisely the singular behavior as we approach the light-like limit. If only two points were becoming light-like separated this limit would be a light-cone operator product expansion, which is a well explored subject, see e.g. the review [37]. Of course, the basic physics is simple, we have a fast moving particle going between the different vertices of the polygon. This fast moving particle is colored and gives rise to a Wilson loop. In section 2.2 this particle was moving so fast that its momentum was above the UV regularization scale, so it behaved like an ordinary free particle. In the present case it behaves as a more complex object. In fact, it behaves as a particle that has a color flux tube attached to it. Since the particle is in the adjoint, this leads in the planar approximation to two copies of the Wilson loop (one in the fundamental and one in the anti-fundamental).

The approach to the limit is determined by the symmetries of the theory. For this reason it is convenient to focus clearly on the symmetries. The symmetries are those of a Wilson loop with a cusp. There are two relevant non-compact symmetries: the boost centered at the cusp and the dilatation also centered at the cusp. These two symmetries commute. They can be made manifest by choosing appropriate coordinates and an appropriate conformal frame, see [38]. We write the original metric as $d s^{2}=d x^{+} d x^{-}+d r^{2}+r^{2} d \varphi^{2}$. We then multiply it by an overall Weyl factor $1 / r^{2}$ to get the metric of $A d S_{3} \times S^{1}$. Then in this $A d S_{3}$ subspace we choose coordinates

$$
\begin{aligned}
d s_{R^{1,3}}^{2} & =\Omega^{2}\left(d s_{A d S_{3}}^{2}+d \varphi^{2}\right), \quad \Omega=r, \\
d s_{A d S_{3}}^{2} & =d \tau^{2}+d \sigma^{2}+2 d \tau d \sigma \sin 2 \beta-d \beta^{2} .
\end{aligned}
$$

Let us consider first a configuration that can give rise to the square Wilson loop. We choose four points sitting at $\tau= \pm \tau_{0}$ and $\sigma= \pm \sigma_{0}$ and $\varphi=0, \beta=0$. These are four spacelike separated points. As $\tau_{0}, \sigma_{0} \rightarrow \infty$ the points approach the vertices of a square light-like polygon. Naively, from the metric in (4.2) one finds that the points are moving infinitely far away. However, due to the conformal factor $\Omega$ that we have introduced in going from $R^{1,3}$ to the metric in (4.2) the points are actually getting closer in the $R^{1,3}$ metric. Thus, the coordinates in (4.2) resolve the region that was giving rise to the divergence. In fact, they have transformed the UV singularity into an IR singularity. Of course, this is a common occurrence in conformal field theories. It is useful to compute the propagator for a field of 
dimension one in these coordinates. This can be done by writing the general propagator as $\langle\phi(1) \phi(2)\rangle=G(1) G(2) /\left(Z_{1} \cdot Z_{2}\right)$ where $Z$ are the coordinates of the projective light-cone in $R^{2,4}$, which obey $Z^{2}=0$ and $Z \sim \lambda Z$. $G$ is a function of the $Z$ 's of homogeneous degree one. Then the above correlator is the one that one should use if we "gauge fix" the symmetry of rescaling of $Z$ 's by setting $G=1$. For example, the ordinary $R^{1,3}$ coordinates are obtained by taking $G=Z^{-1}+Z^{4}$ and $x^{\mu}=Z^{\mu} / G$, with $\mu=0,1,2,3$. The coordinates in (4.2) are obtained by taking $\tilde{G}=\sqrt{\left(Z^{3}\right)^{2}+\left(Z^{4}\right)^{2}}$. Then the coordinates in $A d S_{3}$ correspond to those on the hyperboloid $Y^{2}=-1, Y^{\alpha}=Z^{\alpha} / \tilde{G}$, (with $\alpha=-1,0,1,2$ ), see appendix A for more details. The propagator in the new coordinates takes the form

$$
\Omega(1) \Omega(2)\langle\phi(1) \phi(2)\rangle_{R^{1,3}}=\langle\phi(1) \phi(2)\rangle_{A d S_{3} \times S^{1}}=\frac{1}{2 \cosh \tau_{12} \cosh \sigma_{12}-2},
$$

where we evaluated it at $\beta_{i}=\varphi_{i}=0$. At these points one also finds that

$$
\Omega=\frac{\tilde{G}}{G}=r=\frac{1}{\cosh \tau \cosh \sigma}, \quad \beta=\varphi=0 .
$$

Since the $R^{1,3}$ correlator is inversely proportional to the square of the distance we can also use (4.3) to convert distances into expressions in the new coordinates.

Any four points that are space-like separated can be mapped via a conformal transformation to four points which are at the vertices of a rectangle in the $\tau, \sigma$ plane at $\beta=\varphi=0$ (see figure 6). We can use any coordinate systems to compute the cross ratios of these points. We find that the cross ratios are

$$
\begin{aligned}
|z|^{2} & =\frac{x_{A D}^{2} x_{B C}^{2}}{x_{A C}^{2} x_{D B}^{2}}=\left(\frac{1-\cosh \Delta \tau}{1-\cosh \Delta \sigma \cosh \Delta \tau}\right)^{2} \propto e^{-2 \Delta \sigma}, \\
|1-z|^{2} & =\frac{x_{A B}^{2} x_{D C}^{2}}{x_{A C}^{2} x_{D B}^{2}}=\left(\frac{1-\cosh \Delta \sigma}{1-\cosh \Delta \sigma \cosh \Delta \tau}\right)^{2} \propto e^{-2 \Delta \tau},
\end{aligned}
$$

where $\Delta \tau$ and $\Delta \sigma$ are the sizes of the rectangle. We assumed that both are large $\Delta \tau, \Delta \sigma \gg$ 1 , but their ratio $\Delta \tau / \Delta \sigma$ is arbitrary. The corrections to the above approximation are in powers of the cross ratios that are becoming small and can be neglected for what we will do below.

We see that as we take $\tau_{12} \rightarrow \infty$ in (4.3) the propagator scales as $e^{-\left|\tau_{12}\right|} / \cosh \sigma_{12}$. The fact that it factorizes into a function of $\tau$ and a function of $\sigma$ implies that the energies are independent of the momentum. Here we are defining an energy generator by $\partial_{\tau}$ and a momentum by $-i \partial_{\sigma} \cdot{ }^{11}$ These two operators correspond in the conformal algebra to the operators $D \pm M_{+-}$so that for the particle moving in the ' + ' direction one of them measures the twist and the other the conformal spin. For the particle moving in the '-' direction it is the other way around. This is a result in the free theory. Thus the leading result in the free theory comes from the free propagation of the particles indicated by lines in figure 6(b). This is modified as we go to the interacting theory. Extra singular terms arise precisely due to this modification. Of course, thanks to the factors of $\Omega$ in translating

\footnotetext{
${ }^{11}$ It turns out that this is the same as saying that the twist of a field is independent of the spin, where the twist is defined as $\Delta-S$.
} 


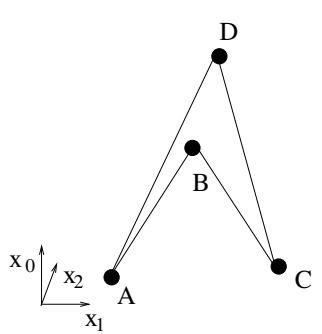

(a)

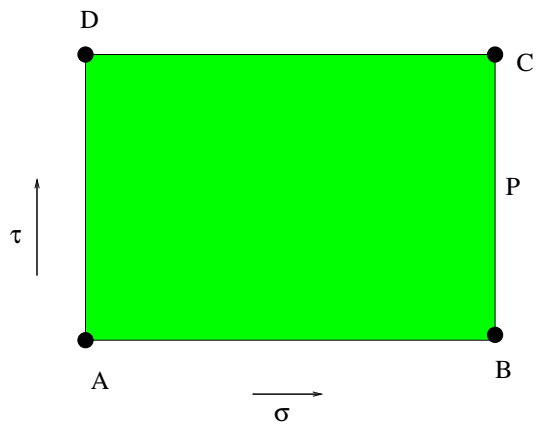

(c)

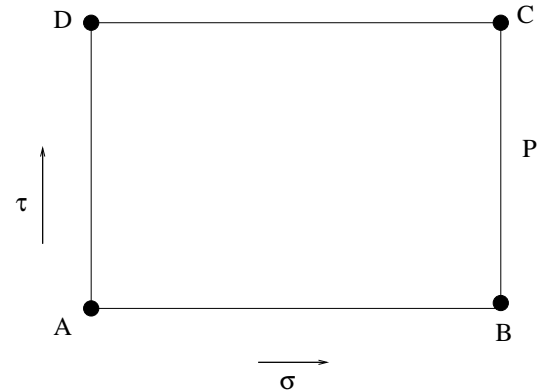

(b)

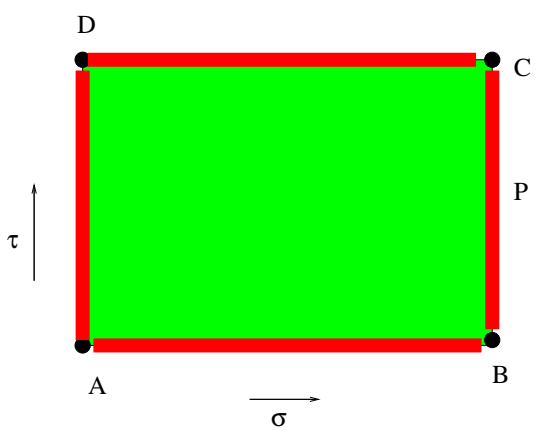

(d)

Figure 6. In (a) we see a polygon with nearly light-like lines. The dots indicate the insertion points of the operators and the lines represent the propagation of a field. In (b) we see the same in the coordinates introduced in (4.2). The points form a rectangle and the light-like limit corresponds to making the rectangle bigger and bigger. The lines represent the propagation of a fast moving particle in the tree level theory. (c) The particles source a color electric field which is extended on the square. (d) This color electric field modifies the propagation of the particles in the full theory. The red lines represent this modification which happens locally at each line.

back to the original coordinates, we get the usual $1 / x_{i, i+1}^{2}$ singularities in the limit. The factors of $\Omega$ are determined by the dimensions of the external operators. For the half-BPS operators of dimension two (3.1), these factors are fixed once and for all and are used to produce the tree level answer.

What are the modifications in the interacting theory? The first and most important modification is due to the fact that the propagating particles carry color charge in the adjoint representation. They create a color flux in the adjoint that goes between the four lines in figure 6(c). This color electric flux has a constant energy density in the $(\tau, \sigma)$-plane. This is not obvious from what we said so far. It becomes more clear if we choose a Lorentzian picture and continue $\tau \rightarrow i t, \quad \beta \rightarrow i \hat{\beta}$. In this case we have the two time-like lines that source the electric field. We consider the situation where these two lines are at a very big distance in the $\sigma$-direction. The final result of the analysis in [38] is that for large $t$ and $\sigma$ one should think of the dynamics as happening in the two dimensions spanned by $t, \sigma$ and the flux has constant energy density. The reason is the following. The $\varphi$ direction is a circle and fields can be KK reduced and at long distances 
only the constant modes would be relevant. The $\hat{\beta}$ direction is non-compact, but there is effectively a gravitational potential which confines the electric flux along this direction. ${ }^{12}$ The effects of the color electric field produce a factor which is proportional to the area of the rectangle in figure 6 (c). The proportionality constant is simply the cusp anomalous dimension $\Gamma_{\text {cusp }}^{\text {adj }}{ }^{13}$ see [38]. In the planar approximation $\Gamma_{\text {cusp }}^{\text {adj }}=2 \Gamma_{\text {cusp }}$, where $\Gamma_{\text {cusp }}$ is the energy density of a flux in the fundamental. Thus we get a contribution of the form $\Gamma_{\text {cusp }}^{\text {adj }} \Delta \tau \Delta \sigma$. Using (4.5) this can be reexpressed as

$$
\Gamma_{\text {cusp }}^{\text {adj }} \Delta \tau \Delta \sigma \sim \frac{\Gamma_{\text {cusp }}^{\text {adj }}}{4} \log |z|^{2} \log |1-z|^{2} \sim \frac{\Gamma_{\text {cusp }}^{\text {adj }}}{4} \log z \log (1-\bar{z}) .
$$

Note that in the light-cone limit we have $z \rightarrow 0$ and $\bar{z} \rightarrow 1 .{ }^{14}$ In general, when two consecutive lines are becoming light-like we can see that there will be a factor of the form $\log x_{12}^{2} \log x_{23}^{2}$ from this reasoning. So far, we are getting a divergent factor of the form

$$
\langle O(1) \cdots O(n)\rangle \longrightarrow e^{-\frac{\Gamma_{\text {cusp }}^{\text {adj }}}{4} \sum_{i=1}^{n} \log \frac{x_{i-1, i}^{2}}{x_{i-1, i+1}^{2}} \log \frac{x_{i, i+1}^{2}}{x_{i-1, i+1}^{2}}} .
$$

This is the most important factor, the rest of the terms involve essentially single logs.

Let us now turn to the second effect that is related to the modification of the propagation of the particles in figure $6(\mathrm{~d})$ due to their interaction with the color flux. There are two sources of corrections. The first is that the energy of the particles can be modified. Instead of being precisely 1, it can be slightly bigger or smaller. If that were the only effect, it would be very easy to take it into account. One would need to change the propagator $e^{-\Delta \tau_{i, i+1}}$ to $e^{-(1+\tilde{g}) \Delta \tau_{i, i+1}}$. Using the formulas in (4.5) applied to points $i-1, i, i+1, i+2$ we find that

$$
\Delta \tau_{i, i+1} \sim-\frac{1}{2} \log \frac{x_{i+1, i+2}^{2} x_{i-1, i}^{2}}{x_{i, i+2}^{2} x_{i-1, i+1}^{2}} \equiv-\frac{1}{2} \log u_{i, i+1} .
$$

We defined the cross ratio $u_{i, i+1}$ which measures the displacement in the $(\tau, \sigma)$-plane between two consecutive points, see figure 7 . This leads to an extra factor of the form

$$
e^{\sum_{i=1}^{n} \frac{\tilde{g}}{2} \log u_{i, i+1}}
$$

where we used that all the particles that are exchanged have identical energy shifts. If we were exchanging uncolored, gauge invariant states, this would be the only effect. However, in our case, the particles also have an electric flux attached to them. Thus, they behave as particles subject to a constant force, and we expect them to "bend" in the presence of this force. The details of this bending depend on the modifications to the dispersion relation, etc. However, we do not need to work them out explicitly for our purposes. For example,

\footnotetext{
${ }^{12}$ The energy conjugate to $t$ can be viewed as the twist $\Delta-S$ when we analyze high spin operators or operators defined along a light-like direction.

${ }^{13} \mathrm{We}$ are normalizing $\Gamma_{\text {cusp }}^{\mathrm{adj}}$ to be the energy density in the $\tau, \sigma$ plane for a flux in the adjoint. One might be worried that the flux in the adjoint would be screened. In the planar approximation it is not. Also in perturbation theory around weak coupling it is not screened, even if we are not in the planar approximation.

${ }^{14}$ Note that $z$ and $\bar{z}$ are complex conjugates in the Euclidean theory but they can be both real in the lorentzian theory.
} 

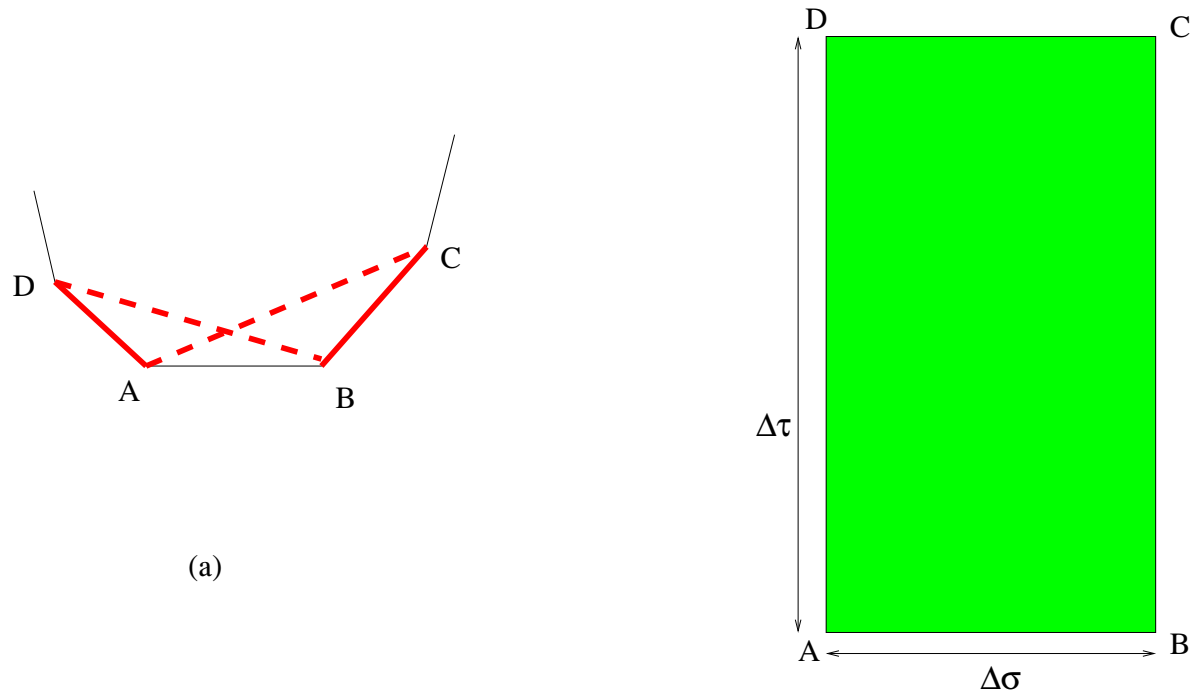

(b)

Figure 7. If we concentrate on points $x_{i}$ and $x_{i+1}$ (here denoted by $A$ and $B$ ) then we see that their distance in the $\tau, \sigma$ plane is given by $\Delta \sigma$ and this is given by the cross ratio $u_{i, i+1}$ represented graphically in (a). Red distances appear in the cross ratio. Solid lines in the numerator and doted lines in the denominator.

let us consider the particle labeled by $\mathrm{P}$ in figure 6 . It moves along the $\tau$ direction. It would not move along the $\sigma$-direction if there were no electric field. In the free theory the particle has a discrete spectrum of energies (or twists), $E=1,2$. These values of the twist correspond to different excitations in the theory. For example, a scalar or some components of the gauge fields have twist one, etc. Let us concentrate on the particle of energy $E=1$, which is the dominant one in our limit. It is important to note that even though the energies are discrete, there is an infinite degeneracy at each energy level. One can label this degeneracy in different ways. For example, we can label the particle by its momentum along $\sigma$. The energy is independent from the momentum in the free theory. As we add the electric field we break the degeneracy. It is useful to understand how this degeneracy is broken. Let us write the energy as $E=1+\epsilon$. With enough patience we could find the energy eigenfunctions $\psi_{\epsilon}(\sigma)$. The important observation is that the original translation symmetry of the problem plus the fact that the electric field is a constant allow us to deduce that the wavefunctions for different energies are all related to each other

$$
\psi_{\epsilon}(\sigma)=\psi_{0}\left(\sigma-\frac{\epsilon}{\Gamma_{\text {cusp }}^{\mathrm{adj}}}\right)
$$

This is a fact that might be familiar to readers who have found the energy eigenstates for a particle in a constant gravitational field. A shift in $\sigma$ translates, through the electric field, into a shift in the energy. We can wonder about the choice of origin in $\sigma$. The electric field is to the left of the particle $P$, see figure 8 . Of course this shift of origin will just lead to constant shift in the energy, or a shift in $\tilde{g}$ in (4.9). Also, when we took into account the 


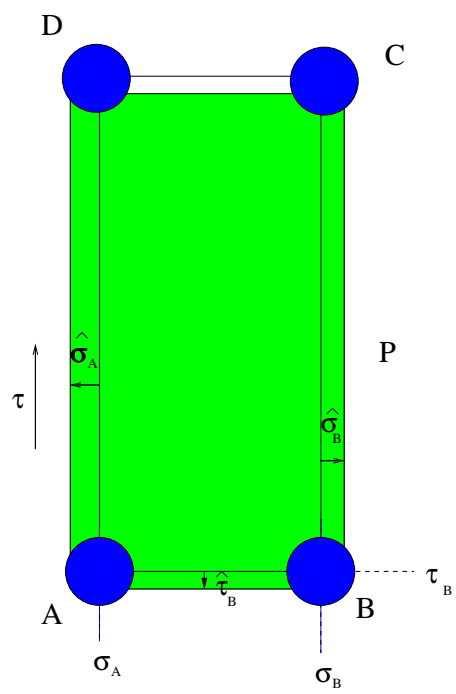

Figure 8. Each operator creates states with a range of energies. A state with a given energy is represented a a straight line which extends up to a new location. For example, the operator $B$ is inserted at $\sigma_{B}$, but the vertical line corresponding to particle $P$ extends to $\sigma=\sigma_{B}+\hat{\sigma}_{B}$. This extent in the $\sigma$ direction is just a nemonic device that tells us what the energy of the state is. There are similar particle propagating from $A$ to $B$ characterized by $\hat{\tau}$, etc.

leading effects of the color electric field, we have cut off its contribution at the position of the insertions of the operator. Thus, we should use this value of $\sigma$ as an origin, and also to set the zero of the energy.

Small constant shifts lead to the terms linear in $\tau$ already taken into account in (4.9). However, the fact that the operator produces a state with a range of energies produces a new effect which we now describe.

Let us consider the particle $P$ that propagates between points $B$ and $C$ in figure 8 . We denote by $\hat{\sigma}_{B}$ the deviation of $\sigma$ from the position of the insertion of the operator which we take at $\sigma=\sigma_{B}, \hat{\sigma}_{B}=\sigma-\sigma_{B}$. Instead of labeling the intermediate states by their energies we use $\hat{\sigma}_{B}$ as a label. The advantage is that we can think of the total energy of the configuration as $\Gamma_{\text {cusp }}^{\mathrm{adj}} \hat{\sigma}_{B}$, where again we have subtracted the part of the flux energy that we have already taken into account. Thus we can label the wavefunction as $\psi_{\hat{\sigma}}\left(\hat{\sigma}^{\prime}\right)=\psi_{0}\left(\hat{\sigma}^{\prime}-\hat{\sigma}\right)$. We will be evaluating this wavefunction at $\hat{\sigma}^{\prime}=0$ which is the insertion point of the operator. Thus we simply get $\psi_{0}(-\hat{\sigma})$. The operator creates a pair of particles with a range of energies. For the operator at $B$, one of them propagates in the $\tau$ direction, towards $A$ and the other in the $\sigma$ direction towards $C$. We denote by $C_{B}\left(\hat{\sigma}_{B}, \hat{\tau}_{B}\right)$ the amplitude that the operator creates a pair of particles with the corresponding energies, labeled here by $\hat{\sigma}_{B}$ and $\hat{\tau}_{B}$, see figure 8 . There is a similar factor for the operator sitting at point $C$. Thus we will get a contribution which goes as

$$
\int d \hat{\sigma} C_{C}\left(\hat{\sigma}, \hat{\tau}_{C}\right) C_{B}\left(\hat{\sigma}, \hat{\tau}_{B}\right) e^{-\Gamma_{\text {cusp }}^{\mathrm{adj}}\left|\tau_{B C}\right| \hat{\sigma}} \psi_{0}(-\hat{\sigma}) \psi_{0}^{*}(-\hat{\sigma}) .
$$

This formula can be interpreted as the expansion of the transition amplitude for a scalar particle to go from point $\mathrm{B}$ to $\mathrm{C}$ in terms of energy (and conformal spin) eigenstates. Here 
we have set $\hat{\sigma}_{B}=\hat{\sigma}_{C}=\hat{\sigma}$ since these are energy eigenstates. Note that the only term that is large here is $\tau_{B C}$ which is diverging in the light-cone limit. There are similar terms involving the propagation of the other particles.

For the case of the square, the final contribution from all such factors has the form

$$
\begin{aligned}
J= & \int d \hat{\sigma} d \hat{\sigma}^{\prime} d \hat{\tau} d \hat{\tau}^{\prime} C_{A}(\hat{\sigma}, \hat{\tau}) C_{D}\left(\hat{\sigma}, \hat{\tau}^{\prime}\right) C_{B}\left(\hat{\sigma}^{\prime}, \hat{\tau}^{\prime}\right) C_{C}\left(\hat{\sigma}^{\prime}, \hat{\tau}^{\prime}\right) \\
& \times e^{-\Gamma_{\text {cusp }}^{\text {adj }}\left|\tau_{A D}\right| \hat{\sigma}} e^{-\Gamma_{\text {cusp }}^{\text {adj }}\left|\sigma_{A B}\right| \hat{\tau}} e^{-\Gamma_{\text {cusp }}^{\text {adj }}\left|\tau_{B C}\right| \hat{\sigma}^{\prime}} e^{-\Gamma_{\text {cusp }}^{\text {adj }}\left|\sigma_{D C}\right| \hat{\tau}^{\prime}} \\
& \times \psi_{0}(-\sigma) \psi_{0}^{*}(-\sigma) \psi_{0}\left(-\sigma^{\prime}\right) \psi_{0}^{*}\left(-\sigma^{\prime}\right) \psi_{0}(-\tau) \psi_{0}^{*}(-\tau) \psi_{0}\left(-\tau^{\prime}\right) \psi_{0}^{*}\left(-\tau^{\prime}\right) .
\end{aligned}
$$

In deriving this relation we have only assumed very general symmetry properties of the theory. Thus, we expect that it should continue to be true for all values of the coupling. We did assume that we created a state that is characterized by a single label, the energy, or equivalently $\hat{\sigma}$. This is definitely true for the creation of twist one excitations in the perturbative theory. This is what is created by an operator (3.3) that has two fields. The coefficients $C(\hat{\sigma}, \hat{\tau})$ depend on the details of the theory and we do not have any prescription for computing them in general. We can replace in (4.12) the distances in the $(\tau, \sigma)$-plane by $u_{i, i+1}$ using (4.8).

The extra factor $J$ looks complicated. However, it only starts contributing at two loops. We will see that for two loops we can actually compute explicitly the functions appearing in it by relating them to the functions that appear in the ordinary operator product expansion. We will do the explicit comparison in section 4.2.

To summarize our discussion, the correlation function in the light-cone limit that we are considering takes the following form

$$
\frac{G_{n}}{G_{n}^{\text {tree }}}=e^{-\frac{\Gamma_{\text {ccusp }}^{\text {adj }}}{4} \sum_{i=1}^{n} \log \frac{x_{i-1, i}^{2}}{x_{i-1, i+1}^{2}} \log \frac{x_{i, i+1}^{2}}{x_{i-1, i+1}^{2}}+\frac{\tilde{g}(\lambda)}{2} \sum_{i=1}^{n} \log u_{i, i+1}} J W_{\text {ren }}^{\text {adj }},
$$

with

$$
J=\int \prod_{i=1}^{n} d \hat{\sigma}_{i} C_{i}\left(\hat{\sigma}_{i}, \hat{\sigma}_{i+1}\right)\left|\psi_{0}\left(-\hat{\sigma}_{i}\right)\right|^{2} e^{\frac{1}{2} \Gamma_{\text {cusp }}^{\text {adj }} \sum_{i=1}^{n} \hat{\sigma}_{i} \log u_{i, i+1}},
$$

where we denoted $\hat{\tau}_{i}$ by $\hat{\sigma}_{i}$ to simplify the notation. Recall that $u_{i, i+1}$ vanish in the same light-cone limit. Here $W_{\text {ren }}^{\text {adj }}$ is the renormalized Wilson loop in the adjoint. It is renormalized by cutting off the divergencies in a way that depends on the distances $x_{i, i+1}^{2}$ not being quite light-like and subtracting the terms that appear multiplying in (4.13). Here we are assuming that we have BPS operators of dimension two that are not renormalized. This is a very particular way of regularizing the Wilson loop. In order to compare to other ways of regularizing the Wilson loop we need to understand how to translate to a different regularization. We will do this translation though a rather indirect route that works nicely when the number of points, $n$, is not a multiple of four. We leave the case where $n$ is a multiple of four to the future.

Let us start from the light-like Wilson loop in dimensional regularization, for example. Then we can write the result as

$$
W^{\text {adj }}=e^{\text {Div }} W_{\text {finite }}^{\text {adj }}, \quad W_{\text {finite }}^{\text {adj }}=e^{\Gamma_{\text {cusp }}^{\text {adj }} A_{\text {BDS-like }}} W_{\text {conformal }},
$$


where the divergent part is given by

$$
\operatorname{Div}=-\sum_{i=1}^{n} \frac{\Gamma_{\text {cusp }}^{\text {adj }}}{8}\left(\log \left(\mu^{2} x_{i, i+2}^{2}\right)\right)^{2}+g \log \left(\mu^{2} x_{i, i+2}^{2}\right)+[\text { poles }],
$$

and [poles] denotes terms involving simple and double poles in $\epsilon$. The finite piece $W_{\text {finite }}^{\text {adj }}$ obeys the Ward identities for anomalous special conformal symmetries [15]. If $n$ is not a multiple of four, we can construct a unique solution of the conformal Ward identities that involves only next to nearest neighbor distances, $x_{i, i+2}^{2}$. This unique expression is what we called $A_{\mathrm{BDS}-\text { like }}$ in (4.15). Its explicit form can be found in formula (A.7) of [39]. ${ }^{15}$ Then the rest, which we called here $W_{\text {conformal }}$, is a conformal invariant function of $x$ 's.

Now, we can do the same trick in the above discussion. One aspect that was not particularly nice in the above discussion was the fact that we had explicit distances appearing in the formulas. In fact, if we factor out the tree expression (4.13), the correlator becomes conformal invariant. Thus, it seems ugly to break the conformal invariance in order to extract the Wilson loop. For this reason, it is convenient to preserve conformal invariance throughout the computation. This can be achieved as follows. First let us understand in more detail what happens as one of the distances $x_{i, i+1}^{2}$ goes to zero while holding the rest small but fixed. In that case we can map the four points $i-1, i, i+1, i+2$ to the vertices of a rectangle in $\tau, \sigma$ plane, see figure 7 . Then the limit $x_{i, i+1}^{2} \rightarrow 0$ corresponds to $\Delta \tau \rightarrow \infty$ and the area grows as

$$
\log G_{n} \rightarrow \Gamma_{\text {cusp }}^{\text {adj }} \Delta \tau \Delta \sigma=\frac{\Gamma_{\text {cusp }}^{\text {adj }}}{4} \log x_{i, i+1}^{2} \log u_{i, i+1},
$$

where we used (4.8). This is valid up to terms that remain finite when $x_{i, i+1}^{2}$ goes to zero. Of course, the expression (4.7) obeys (4.17). We now would like to write an expression which involves only cross ratios which also obeys (4.17). This is easy to do when $n$ is not a multiple of four. In that case, a given distance $x_{i, i+1}^{2} \rightarrow 0$ can be promoted to a unique cross-ratio (if $n \neq 4 k$ ) that involves the distance $x_{i, i+1}^{2}$ and next-to-neighboring distances of the form $x_{j, j+2}^{2}$. We denote by $\chi_{i, i+1}$ this unique cross-ratio. Then one also finds that $u_{i, i+1}=\chi_{i-1, i} \chi_{i+1, i+2}$. Thus each cusp gives rise to a term $-\frac{\Gamma_{\text {cusp }}^{\text {adj }}}{4} \log \chi_{i-1, i} \log \chi_{i, i+1}$. Then we can rewrite the limit as ${ }^{16}$

$$
\begin{aligned}
\frac{\langle O(1) \cdots O(n)\rangle}{\langle O(1) \cdots O(n)\rangle_{\text {tree }}} & =\mathcal{F}(\text { cross-ratios }) \\
& =e^{-\frac{\Gamma_{\text {cusp }}^{\text {adj }}}{4} \sum_{i=1}^{n} \log \chi_{i-1, i} \log \chi_{i, i+1}+\frac{\tilde{g}(\lambda)}{2} \sum_{i=1}^{n} \log u_{i, i+1}} J W_{\text {conformal }}^{\text {adj }}
\end{aligned}
$$

In writing (4.18) we have asserted that $W_{\text {conformal }}$ is indeed the same as $W_{\text {conformal }}$ in (4.15). More precisely, in a planar theory we would get $W_{\text {conformal }}^{\text {adj }}=\left(W_{\text {conformal }}\right)^{2}$, where $W_{\text {conformal }}$ is the Wilson loop in the fundamental. The reason is that both are a renormalized version

\footnotetext{
${ }^{15}$ Actually, they differ by a factor of two and a sign $A_{\mathrm{BDS}-\text { like }}^{\text {here }}=-2 A_{\mathrm{BDS}-\text { like }}^{\text {in }}$.

${ }^{16}$ Here we assumed that the operators are BPS so that dividing by the tree correlator we get a conformal invariant answer.
} 
of the Wilson loop which has been made conformal invariant by the use of next-to-nearest neighboring distances. Thus we expect that both should coincide. In the special case of the four point function, $n=4$, the expression (4.18) continues to be valid after replacing $\chi_{12} \chi_{34}=u_{2,3}=|z|^{2}, \chi_{23} \chi_{41}=u_{34}=|1-z|^{2}$. The cases where $n$ is other multiples of four is left for the future.

In fact, we will check below that the two expressions for $W_{\text {conformal }}$ coincide at one loop. At one loop the factor $J$ can be set to one. The reason is that any single logarithm appearing in $J$ can be absorbed in a redefinition of the function $\tilde{g}$. Only at two loops we expect to see the appearance of the factor $J$. In fact, we consider the four point correlation function (which is the only available full correlator at two loops) and we check the appearance of terms that can be interpreted as arising from the factor $J$. We will perform these two checks in the next two subsections.

The attentive reader might be surprised by a certain factor of two for the leading double logarithmic terms in the expression for the correlation function $G_{n}$, eq. (4.7), and light-like Wilson loop in the adjoint representation $W^{\text {adj }}$, eq. (4.16). Namely, if we set the distances $x_{i, i+1}^{2}=\mu^{-2}$ then the coefficient in front of double logs in the two expressions differ by a factor of two. The origin of this factor of two is explained in appendix D. (This two is unrelated to the one that arises when we go from the adjoint to two copies of the fundamental in the planar limit.)

\subsection{One-loop checks}

In this subsection we check by an explicit one-loop calculation that the light-cone limit of the $n$-point correlators (3.4) has the form (4.18), and evaluate the one-loop expression for $W_{\text {conformal }}$. As we mentioned above, the factor $J$ can be set to 1 at one loop.

The one-loop correlators of BPS operators were computed in [34, 35, 40-42]. In our limit we get

$$
\begin{aligned}
\lim _{x_{i, i+1}^{2} \rightarrow 0}\left(G_{n} / G_{n}^{\text {tree }}\right) & =-\frac{i a}{4 \pi^{2}} \lim _{x_{i, i+1}^{2} \rightarrow 0} \sum_{k, l} \int d^{4} x_{0} \frac{x_{k, l+1}^{2} x_{k+1, l}^{2}-x_{k l}^{2} x_{k+1, l+1}^{2}}{x_{k, 0}^{2} x_{k+1,0}^{2} x_{l, 0}^{2} x_{l+1,0}^{2}} \\
& =-\frac{a}{2} \lim _{x_{i, i+1}^{2} \rightarrow 0} \sum_{k, l}\left(x_{k, l+1}^{2} x_{k+1, l}^{2}-x_{k l}^{2} x_{k+1, l+1}^{2}\right) g(k, k+1, l, l+1)
\end{aligned}
$$

where $a=g^{2} N_{c} /\left(8 \pi^{2}\right)$ is the 't Hooft coupling constant, and the integral $g(1,2,3,4)$ (the dual space version of the one-loop scalar box) is defined as

$$
g(1,2,3,4)=\frac{i}{2 \pi^{2}} \int \frac{d^{4} x_{0}}{x_{10}^{2} x_{20}^{2} x_{30}^{2} x_{40}^{2}} .
$$

As long as the outer points are kept in generic positions, $x_{i, i+1}^{2} \neq 0$, this integral is finite and conformally covariant in $D=4$ dimensions. This allows us to write it down as a function of two conformal cross-ratios

$$
g(1,2,3,4)=\frac{1}{x_{13}^{2} x_{24}^{2}} \Phi^{(1)}(x, y), \quad \text { with } \quad x=\frac{x_{14}^{2} x_{23}^{2}}{x_{13}^{2} x_{24}^{2}}, \quad y=\frac{x_{12}^{2} x_{34}^{2}}{x_{13}^{2} x_{24}^{2}},
$$


where the two-variable function $\Phi^{(1)}$ is given by $[43,44]$

$$
\Phi^{(1)}(x, y)=\frac{1}{2} \int_{0}^{1} d \xi \frac{\log (y / x)+2 \log \xi}{y \xi^{2}+(1-x-y) \xi+x} .
$$

Let us now examine the light-cone limit $x_{i, i+1}^{2} \rightarrow 0$. In eq. (4.19) the arguments of the $g$ integrals are pairwise adjacent, $\left(x_{k}, x_{k+1}\right)$ and $\left(x_{l}, x_{l+1}\right)$. This implies that, in the light-cone limit $x_{i, i+1}^{2} \rightarrow 0$, at least one of the two cross-ratios on which the $g$-integral depends goes to zero in this limit. If in addition $l=k \pm 2$, all points are adjacent, in which case both cross-ratios tend to zero. The leading singular behavior of $\Phi^{(1)}(x, y)$ when $y \rightarrow 0$ is given by

$$
\begin{aligned}
\lim _{y \rightarrow 0} \Phi^{(1)}(x, y) & =\frac{1}{2} \int_{0}^{1} d \xi \frac{\log (y / x)+2 \log \xi}{(1-x) \xi+x}+O(y) \\
& =-\frac{1}{2(1-x)}\left[\log x \log y-2 \operatorname{Li}_{2}\left(1-\frac{1}{x}\right)-\log ^{2}(x)\right]+O(y) .
\end{aligned}
$$

We will need (4.23) for $n \geq 6$, while for $n=4,5$ the double limit $x, y \rightarrow 0$ is relevant:

$$
\lim _{x, y \rightarrow 0} \Phi^{(1)}(x, y)=-\frac{1}{2} \log x \log y-\frac{\pi^{2}}{6}+O(x)+O(y) .
$$

In the simplest case of four points we have

$$
\begin{aligned}
\lim _{x_{i, i+1}^{2} \rightarrow 0}\left(G_{4} / G_{4}^{\text {tree }}\right) & =2 a x_{13}^{2} x_{24}^{2} \lim _{x_{i, i+1}^{2} \rightarrow 0} g(1,2,3,4) \\
& =-a\left[\log x \log y+\frac{\pi^{2}}{3}\right]+O(x)+O(y) .
\end{aligned}
$$

If we put

$$
x_{i, i+1}^{2}=1 / \epsilon_{c}^{2} \rightarrow 0,
$$

where $\epsilon_{c}$ is the regulator from appendix $\mathrm{A}$ in [39], we obtain

$$
\begin{aligned}
\lim _{x_{i, i+1}^{2} \rightarrow 0}\left(G_{4} / G_{4}^{\text {tree }}\right) & =-a\left[8 A_{\mathrm{div}}-\log ^{2}\left(\frac{x_{13}^{2}}{x_{24}^{2}}\right)+\frac{\pi^{2}}{3}\right] \\
& =-a\left[8 A_{\mathrm{div}}-2 A_{\mathrm{BDS}}+4 \times \frac{5}{2} \zeta(2)\right],
\end{aligned}
$$

where $A_{\text {div }}$ is defined in equation (A.6) in [39] and at four points $A_{\mathrm{BDS}}$ is essentially the $\log ^{2}$ term. ${ }^{17}$ This term is finite but obviously not conformally invariant. On the other hand, the correlator $G_{4}$ in $D=4$ is conformal before putting $x_{i, i+1}^{2}=1 / \epsilon_{c}^{2}$; it is a function of crossratios. But we can do better: by adding and subtracting the non-conformal expressions $A_{\mathrm{BDS}}$ from $A_{\mathrm{BDS}-\text { like }}$ (the latter is defined in (A.7) in [39]) and putting it together with the other non-conformal piece $A_{\text {div }}$, we can produce a manifestly conformal "remainder", which is the one-loop value of the factor $W_{\text {conformal }}$ from (4.18). In what follows we confirm this explicitly for $n=5,6,7$ and claim that it works for all $n \neq 4 k$.

\footnotetext{
${ }^{17}$ By definition, $A_{\mathrm{BDS}}$ is the finite part in the $n$-gluon one-loop MHV amplitude and is given in [45].
} 
In the limiting prescription (4.26) we have put all small distances equal. On the other hand, we may ask whether any given small distance $x_{i, i+1}^{2}$ can be uniquely promoted to a cross-ratio using only members of the next-to-nearest neighbor set $\left\{x_{j, j+2}^{2}\right\}$, as is suggested by the form of $A_{\text {div }}$ in (A.7) in [39]. In order to answer this, one simply solves a linear system on the exponents of the $\left\{x_{j, j+2}^{2}\right\}$ in a general ratio, requiring the conformal weight to cancel out at each point. No solution exists when the number of points is $n=4 k$. For all other values of $n$ we find

$$
\begin{aligned}
n=4 k+1: & \chi_{i, i+1}=\frac{x_{i, i+1}^{2} x_{i+2, i+4}^{2} \cdots x_{i-3, i-1}^{2}}{x_{i, i+2}^{2} x_{i+4, i+6}^{2} \cdots x_{i-1, i+1}^{2}} \\
n=4 k+3: & \chi_{i, i+1}=\frac{x_{i, i+1}^{2} x_{i+3, i+5}^{2} \cdots x_{i-4, i-2}^{2}}{x_{i+1, i+3}^{2} x_{i+5, i+7}^{2} \cdots x_{i-2, i}^{2}} \\
n=4 k+2: & \chi_{i, i+1}=\frac{x_{i, i+1}^{2}}{\sqrt{R_{i} R_{i+1}}}, \quad R_{i}=\frac{x_{i, i+2}^{2} x_{i+4, i+6}^{2} \cdots x_{i-2, i}^{2}}{x_{i+2, i+4}^{2} \cdots x_{i-4, i-2}^{2}} .
\end{aligned}
$$

We can now check that at one loop

$$
\lim _{x_{i, i+1}^{2} \rightarrow 0}\left(G_{n} / G_{n}^{\text {tree }}\right)=-a\left[\sum_{i=1}^{n} \log \chi_{i, i+1} \log \chi_{i+1, i+2}-2\left(A_{\mathrm{BDS}}-A_{\mathrm{BDS}-\text { like }}\right)+n \times \frac{5}{2} \zeta(2)\right] .
$$

Since (4.19) contains only $g(k, k+1, l, l+1)$, the arguments of $g$ are always at least pairwise adjacent. This guarantees that the asymptotic expansions (4.23), (4.24) are sufficient to obtain the one-loop correlators for all $n$. The last equation can easily be verified up to rather large values of $n \neq 4 k$.

Explicitly, for $n=5,6,7$ we find the following results for the limit (4.29):

$$
\begin{aligned}
\lim _{x_{i, i+1}^{2} \rightarrow 0} \frac{G_{5}}{G_{5}^{\text {tree }}}=-a & {\left[\sum_{i=1}^{5} \log \chi_{i, i+1} \log \chi_{i+1, i+2}+5 \frac{\pi^{2}}{3}\right], } \\
\lim _{x_{i, i+1}^{2} \rightarrow 0} \frac{G_{6}}{G_{6}^{\text {tree }}}=-a & {\left[\sum_{i=1}^{6} \log \chi_{i, i+1} \log \chi_{i+1, i+2}+6 \frac{\pi^{2}}{3}+\sum_{i=1}^{3}\left(\frac{1}{2} \log ^{2} u_{i}+\operatorname{Li}_{2}\left(1-u_{i}\right)\right)\right], } \\
\lim _{x_{i, i+1}^{2} \rightarrow 0} \frac{G_{7}}{G_{7}^{\text {tree }}}=-a & {\left[\sum_{i=1}^{7} \log \chi_{i, i+1} \log \chi_{i+1, i+2}+7 \frac{\pi^{2}}{3}+\sum_{i=1}^{7}\left(\frac{1}{2} \log ^{2} u_{i}+\operatorname{Li}_{2}\left(1-u_{i}\right)\right)\right.} \\
& \left.+\sum_{j>i} \frac{1}{2} c_{i j} \log u_{i} \log u_{j}\right] .
\end{aligned}
$$

In the last relation the coefficient $c_{i j}$ is equal to:

$$
c_{i j}=0: d(i, j)=1, \quad c_{i j}=-1: d(i, j)=2, \quad c_{i j}=1: d(i, j)=3,
$$

where $d(i, j)$ is the shortest cyclic distance of two corners of the heptagon, so $d(2,7)=2$, etc. The cross-ratios are defined by

$$
u_{i}=\frac{x_{i, i+4}^{2} x_{i+1, i+3}^{2}}{x_{i, i+3}^{2} x_{i+1, i+4}^{2}}
$$


The finite parts in (4.30) are the explicit versions of $-2\left(A_{\mathrm{BDS}}-A_{\mathrm{BDS}-\text { like }}\right)$ for these values of $n$.

\subsection{A two-loop check for the four point correlator}

In this subsection we consider the four-point correlation function at two loops. We take the planar limit and set $\Gamma_{\text {cusp }}^{\mathrm{adj}}=2 \Gamma_{\text {cusp }}$ in all formulas. The two-loop correction to the fourpoint correlation function was computed in [31-33] and it is given by ${ }^{18}$ (with $\lambda=g^{2} N_{c}$ )

$$
\begin{aligned}
\frac{G_{4}}{G_{4}^{\text {tree }}}= & 1-\frac{\lambda}{8 \pi^{2}} \Phi_{1}(z, \bar{z})+\frac{\lambda^{2}}{16 \pi^{4}}\left\{\frac{2+2 z \bar{z}-z-\bar{z}}{16}\left[\Phi_{1}(z, \bar{z})\right]^{2}\right. \\
& \left.+\frac{1}{4(z-\bar{z})}\left(\Phi_{2}(z, \bar{z})-\Phi_{2}(1-z, 1-\bar{z})-\Phi_{2}\left(\frac{z}{z-1}, \frac{\bar{z}}{\bar{z}-1}\right)\right)\right\},
\end{aligned}
$$

where the notation was introduced for

$$
\begin{aligned}
\Phi_{1}(z, \bar{z})= & \frac{1}{z-\bar{z}}\left(2 \operatorname{Li}_{2}(z)-2 \operatorname{Li}_{2}(\bar{z})+\log (z \bar{z}) \log \frac{1-z}{1-\bar{z}}\right) \\
\Phi_{2}(z, \bar{z})= & 6\left(\operatorname{Li}_{4}(z)-\operatorname{Li}_{4}(\bar{z})\right)-3 \log (z \bar{z})\left(\operatorname{Li}_{3}(z)-\operatorname{Li}_{3}(\bar{z})\right)+ \\
& +\frac{1}{2} \log ^{2}(z \bar{z})\left(\operatorname{Li}_{2}(z)-\operatorname{Li}_{2}(\bar{z})\right) .
\end{aligned}
$$

We can check that the logarithm of the ratio (4.33) has the following expansion, up to constant terms, as we take $z \rightarrow 0^{+}$and $\bar{z} \rightarrow 1^{-}$

$$
\begin{aligned}
\log \left(\frac{G_{4}}{G_{4}^{\text {tree }}}\right) \sim & -\frac{\Gamma_{\text {cusp }}}{2} \log z \log (1-\bar{z})-\frac{B}{2}(\log z+\log (1-\bar{z})) \\
& +\frac{\left(2 \Gamma_{\text {cusp }}\right)^{2} \pi^{2}}{96}\left[(\log z)^{2}+(\log (1-\bar{z}))^{2}\right]
\end{aligned}
$$

where

$$
2 \Gamma_{\text {cusp }}=\frac{\lambda}{2 \pi^{2}}-\frac{\lambda^{2}}{96 \pi^{2}}+\cdots, \quad B=\frac{3}{32 \pi^{4}} \zeta_{3} \lambda^{2}+\cdots,
$$

Note that equation (4.36) should be trusted only to second order in $\lambda$.

Let us now compare (4.36) with (4.18). The first line in (4.36) corresponds to the terms in (4.18) involving the cusp anomalous dimension. The second line in (4.36) contains double logarithmic terms which we would like to interpret as coming from the factor $J$ in (4.18). For this purpose, we would like to compute the wave-functions $C(\hat{\sigma}, \hat{\tau})$. Since we will be looking at terms that involve two logs and two loops, it is clear that we only need $C$ at tree level. At tree level the wave function factorizes $C=C_{T}(\hat{\sigma}) C_{T}(\hat{\tau})$, since the operator (3.3) is the product of two fields and each field creates one particle. Thus, we are interested in terms of the form $u(\hat{\sigma}) \equiv\left[C_{T}(\hat{\sigma})\right]^{2} \psi_{0}(-\hat{\sigma}) \psi_{0}^{*}(-\hat{\sigma})$. More explicitly, the $J$ factor (4.14) can be expressed in terms of the Fourier (or Laplace) transform of $u$, which we denote by $v(k)$ :

$$
\begin{aligned}
J & =\left[\int d \hat{\sigma} u(\hat{\sigma}) e^{\hat{\sigma} \Gamma_{\text {cusp }} \log z} \int d \hat{\tau} u(\hat{\tau}) e^{\hat{\tau} \Gamma_{\text {cusp }} \log z}\right]^{2} \\
& =\left[v\left(-i \Gamma_{\text {cusp }} \log z\right) v\left(-i \Gamma_{\text {cusp }} \log (1-\bar{z})\right)\right]^{2}
\end{aligned}
$$

\footnotetext{
${ }^{18}$ The relation between the variables used in the previous subsection and this one is $x=z \bar{z}, y=(1-$ $z)(1-\bar{z})$.
} 
with

$$
v(k) \equiv \int d \hat{\sigma} e^{i k \sigma} u(\hat{\sigma})
$$

The $v$-factors can be computed indirectly by considering a slightly different problem which involves precisely the same functions. Namely, consider the one-loop four-point correlation function and take the light-cone OPE limit corresponding to $z \rightarrow 0^{+}$with $\bar{z}$ kept finite. We then extract the contribution from each given conformal spin to the OPE and take the limit where $\bar{z} \rightarrow 1$. In this limit the spin that contributes most to the OPE is large. We will then make the same sort of approximations that we discussed above to represent the answer. This computation is possible thanks to the expressions in [46], which spell out the contribution of each conformal towers to the OPE.

We expect that the limit $\bar{z} \rightarrow 1$ should be dominated by the large spin contributions to the OPE. For reasons that are completely similar to the ones given above, we expect that the large spin contribution to the OPE for the four-point correlation function should look like

$$
\begin{aligned}
G_{4} & \sim e^{-2 \Gamma_{\text {cusp }} \Delta \tau\left(2 \sigma_{0}\right)} \int d \hat{\sigma}_{1} u\left(\hat{\sigma}_{1}\right) \int d \hat{\sigma}_{2} u\left(\hat{\sigma}_{2}\right) e^{-2 \Gamma_{\text {cusp }} \Delta \tau\left(\hat{\sigma}_{1}+\hat{\sigma}_{2}\right)} \\
& =e^{-2 \Gamma_{\text {cusp }} \Delta \tau\left(2 \sigma_{0}\right)}\left[v\left(i 2 \Gamma_{\text {cusp }} \Delta \tau\right)\right]^{2} \\
& =\int d \sigma_{s} e^{-2 \Gamma_{\text {cusp }} \Delta \tau\left(2 \sigma_{0}+\sigma_{s}\right)} \int d \hat{\sigma}_{1} u\left(\hat{\sigma}_{1}\right) u\left(\sigma_{s}-\hat{\sigma}_{1}\right)
\end{aligned}
$$

where $2 \Delta \tau \sim-\log z, 4 \sigma_{0} \sim-\log (1-\bar{z})$ and $v$ is the Fourier transform of $u$, as in (4.38). This formula is derived as follows. $\tau$ and $\sigma_{0}$ characterize the points where the operators are inserted. The operators create particles at the center of the blue circles in figure 9 . The particle states can be expanded in energy eigenstates for the problem of a particle which has a color electric adjoint flux ending on it. The energy eigenstates can be labeled by a position $\hat{\sigma}_{1}$. The blue circles represent the overlap between these wave functions and the field operator. The filled rectangle in figure 9 represents the energy of the intermediate state. It is proportional to the width of the rectangle and is given by

$$
E=2 \Gamma_{\text {cusp }}\left(2 \sigma_{0}+\sigma_{s}\right), \quad \sigma_{s} \equiv \hat{\sigma}_{1}+\hat{\sigma}_{2} .
$$

Thus, we have essentially the same setup that we were using when we talked about the Wilson loop. In fact, it is the same correlator, except that here we are thinking in terms of an ordinary $\mathrm{OPE}$ and we are only concentrating on the terms going like $e^{-\tau E}$ where $E$ is the twist of the operator. In (4.41) we ignored the possibility of adding a $\hat{\sigma}_{i}$ independent constant. This is included in the function $\tilde{g}$. In conclusion, (4.40) is what we expect for the OPE as $z \rightarrow 0$ with $\bar{z} \sim 1$ from our point of view.

The OPE was analyzed in great detail by Dolan and Osborn in [46]. They considered the asymptotic behavior of the four-point correlator for $z \rightarrow 0$ with $\bar{z}$ fixed, and isolated the contributions from the conformal tower with the conformal spin $S$. Each conformal tower has an anomalous dimension which uniquely specifies it and is given for large spin 


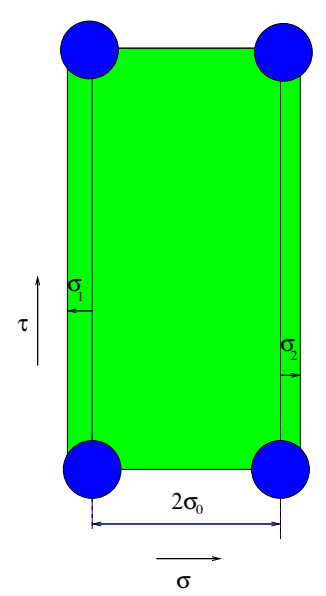

Figure 9. We consider the operator product expansion with $z \rightarrow 0$ and $1-\bar{z}$ very small but fixed. In this regime $\sigma_{0}$ is large but $\Delta \tau$ is going to infinity. Thus the states that propagate in the OPE propagate along the $\tau$ direction in this picture. The width of the rectangle represents the energy of the state. The circles represent the fact that the operator creates states with a range of energies, so that the energy of the intermediate state depends not only on $\sigma_{0}$ but also on $\hat{\sigma}_{1}, \hat{\sigma}_{2}$ which are the extra energies of the propagating particles at the two ends.

by $E=\Delta-S=2 \Gamma_{\text {cusp }} \log S[47,48]$, where we ignored the subleading constant that is independent of the spin. For large $S$ the OPE takes the form

$$
G_{4} \sim \sum_{S} z^{\frac{\Delta-S}{2}} H_{S}(\bar{z})=\sum_{S} z^{\Gamma_{\text {cusp }} \log S} H_{S}(\bar{z})
$$

with $H_{S}$ given in terms of a hypergeometric function,

$$
H_{S}(\bar{z})=\frac{\Gamma(S+2) \Gamma(S+3)}{\Gamma(2 S+4)} \bar{z}^{S} F(a, a, 2 a ; \bar{z}), \quad a=S+3 .
$$

We now compare (4.42) with (4.40). We need to translate between $\bar{z}$ and the spin $S$ and the variables $\sigma_{s}$ and $\sigma_{0}$. From (4.8) we find that $(1-\bar{z}) \propto e^{-4 \sigma_{0}}$. Comparing the term involving the anomalous dimensions (the $z$ dependent term) in (4.42) with (4.40) we find

$$
\log S=2 \sigma_{0}+\sigma_{s}
$$

In the large $S$ limit we can convert the sum over $S$ to an integral and then turning this into an integral over $\sigma_{s}$ we obtain the following measure factor $\sum_{S} \rightarrow \int d S=e^{2 \sigma_{0}} \int e^{\sigma_{s}} d \sigma_{s}$. After multiplying (4.43) by the tree level $(1-\bar{z})$ to remove the tree level singularity we find

$$
\frac{G_{4}}{G_{4}^{\text {tree }}} \sim \lim _{\sigma_{0} \rightarrow \infty}(1-\bar{z}) \sum_{S} z^{\frac{\Delta-S}{2}} H_{S}(\bar{z}) \propto \int d \sigma_{s} z^{\Gamma\left(2 \sigma_{0}+\sigma_{s}\right)} e^{2 \sigma_{s}} K_{0}\left(2 e^{\sigma_{s}}\right),
$$


where we used ${ }^{19}$

$$
(1-\bar{z}) H_{S}(\bar{z}) \propto e^{-2 \sigma_{0}} e^{\sigma_{s}} K_{0}\left(2 e^{\sigma_{s}}\right), \quad \text { for } \quad \sigma_{0} \gg 1
$$

Comparing the $z$ independent part of the integrand in (4.45) with (4.40) we find that

$$
\int d \hat{\sigma}_{1} u\left(\hat{\sigma}_{1}\right) u\left(\sigma_{s}-\hat{\sigma}_{1}\right)=e^{2 \sigma_{s}} K_{0}\left(2 e^{\sigma_{s}}\right)
$$

Fourier transforming on both sides gives

$$
[v(k)]^{2} \propto \int d \sigma_{s} e^{i k \sigma_{s}} e^{2 \sigma_{s}} K_{0}\left(2 e^{\sigma_{s}}\right) \propto\left[\Gamma\left(1+\frac{i k}{2}\right)\right]^{2} .
$$

Returning to our problem, we can now evaluate the factor $J$ in (4.38)

$$
\begin{aligned}
J & \sim\left[\Gamma\left(1+\frac{\Gamma_{\text {cusp }} \log z}{2}\right) \Gamma\left(1+\frac{\Gamma_{\text {cusp }} \log (1-z)}{2}\right)\right]^{2} \\
& =e^{-\gamma_{\mathrm{E}} \Gamma_{\text {cusp }}(\log z+\log (1-z))}\left\{1+\frac{\pi^{2}\left(2 \Gamma_{\text {cusp }}\right)^{2}}{96}\left[(\log z)^{2}+(\log (1-z))^{2}\right]+\cdots\right\}
\end{aligned}
$$

This computation is only trustworthy to second order in $\Gamma_{\text {cusp }}$. The resulting expression for $J$ reproduces precisely double logarithms in the second line of (4.36). The term involving Euler's constant $\gamma_{\mathrm{E}}$ looks like a contribution to the function $\tilde{g}$. At one loop we know that there is no single log. Thus this term must be canceled by a constant contribution to the energy of the particle, which could come from a simple shift in our origin of $\sigma$ when we considered the energy eigenstates. In fact, we can include these constant contributions to $\Delta-S=2 \Gamma_{\text {cusp }}\left(\log S+\gamma_{\mathrm{E}}\right)-B$. There is a finite piece involving $\gamma_{\mathrm{E}}$ which precisely cancels the $\gamma_{\mathrm{E}}$ in (4.49). There is, however a two loop contribution $B$ which precisely matches the two loop logarithm in the first line of (4.36).

Note that the above two-loop computation of $J$ can now be used to write the factor $J$ for any $n$ point correlation function involving the same BPS operators as in the four-point correlator (3.4)

$$
J=\prod_{i=1}^{n} \check{v}\left(\frac{\Gamma_{\text {cusp }}}{2} \log u_{i, i+1}\right), \quad \check{v}(w) \equiv \Gamma(1+w) e^{\gamma_{\mathrm{E}} w} .
$$

Similarly, the function $\tilde{g}$ in (4.18) is equal, up to two loops, to

$$
\tilde{g}=-\frac{B}{2}
$$

\footnotetext{
${ }^{19}$ This limit can be understood as follows. $H_{S}(\bar{z})$ satisfies a simple quadratic differential equation on $\bar{z}$, where $S$ plays the role of a parameter. Making a change of variables $\bar{z}=1-\epsilon, S=\frac{x}{\sqrt{\epsilon}}$ we can see this equation as an equation for $H(\epsilon, x)$. Assuming a small $\epsilon$ behavior of the form $H(\epsilon, x)=\frac{h(x)}{\sqrt{\epsilon}}+\ldots$, which is easy to show, it can be seen that $h(x)$ satisfies a simple differential equation whose solution is $x K_{0}(2 x)$.
} 


\section{Conclusions}

In this paper we have considered a multiple light-like limit of correlation function which leads to Wilson loops. Namely, we started with a correlation function of local operators $\left\langle\mathcal{O}\left(x_{1}\right) \cdots \mathcal{O}\left(x_{n}\right)\right\rangle$ with all points space-like separated. Then we considered the limit where $x_{i, i+1}^{2} \rightarrow 0$ but with $x_{i}^{\mu} \neq x_{i+1}^{\mu}$, so that the points are separated along a null direction. In this limit the operators are sitting at the vertices, or cusps, of a polygon with $n$ null edges. As we approach the limit, the correlator develops a singularity that is due to the exchange of a fast moving particle from one operator to the next. These fast moving particles define the null polygonal frame which is the source of a color electric field. If all particles in the theory are in the adjoint representation, this color electric field is in the adjoint. This procedure allows us to find the Wilson loop with null polygonal edges as a limit of the correlator.

In order to extract the Wilson loop, we needed to isolate the contribution from the fast propagating particles. The easiest way to do this is to dimensionally regularize the correlator and then take the limit where the distances $x_{i, i+1}^{2} \rightarrow 0$, with the regulator scale held fixed. In this limit the particles are propagating in a free theory and their contribution is the same as that of the tree-level correlator in the same limit. Each particle contributes with a simple factor of $1 / x_{i, i+1}^{2}$. Once this factor is extracted from the correlator we get the Wilson loop in dimensional regularization (2.15). Of course, one disadvantage of this approach is that we need to compute the correlators in dimensional regularization. But this is definitely something that one can do. For the particular case of $\mathcal{N}=4 \mathrm{SYM}$ we demonstrated how to do it at one loop.

One can also take the light-like limit purely in the four dimensional theory. In that case the particles that propagate are "dressed" by the interactions and the approach to the limit is a bit more subtle. We have untangled these subtleties and arrived at the relation (4.13) that explains clearly how the limit is approached. The cusp divergences of the Wilson loop appear as $\propto \Gamma_{\text {cusp }} \log x_{i, i+1}^{2} \log x_{i-1, i}^{2}$ terms in the exponential. This is a simple and expected factor. The more subtle terms involve contributions due to the fast propagating particles. The factor of the form $\prod 1 /\left(x_{i, i+1}^{2}\right)^{1+\tilde{g}(\lambda)}$ arises due to corrections to the "energies" of the propagating particles. There is however a more subtle factor that comes from the back reaction of the color electric field on the propagation of the particle. This is the $J$ factor in (4.14). We have computed $J$ explicitly at two loops in (4.49) for the four-point correlator. The extraction of the Wilson loop from the correlation function can be done in a way that preserves conformal symmetry throughout. This is done by extracting the various singular terms via cross ratios. What remains is a renormalized Wilson loop which is explicitly conformally invariant (4.18). The precise expression depends on how we extracted the divergent terms. If we extract them in terms of a special kind of cross-ratios, then the Wilson loop is defined by subtracting a very specific function, called "BDS-like", from the finite piece of the Wilson loop computed in the dimensional regularization. The finite piece of the dimensionally regularized Wilson loop is not conformally invariant but it obeys the anomalous Ward identity [11]. The BDS-like expression [39] is the unique way to solve this anomaly by considering a function of only next-to-nearest neighbor distances, $x_{i, i+2}^{2}$. This procedure works and is well defined when the number of sides is not a multiple of four, $n \neq$ 
$4 k$. We have not treated the $n=4 k$ case here and we leave that for the future. However, we did check that the correlator reproduces the BDS - (BDS-like) expression at one loop.

This basic connection between correlators and polygonal Wilson loops should be true for general conformal gauge theories in any space-time dimensions.

This expansion could also be done for $\mathcal{N}=4$ super-Yang-Mills at strong coupling by using strings in $A d S_{5} \times S^{5}$. We expect it to work in a similar fashion. In fact, a closely related computation was done in appendix B.1 of [49], where the large spin limit of single trace operators was considered.

In planar gauge theories the adjoint Wilson loop can be viewed as a product of a fundamental and an anti-fundamental Wilson loops. In theories with dynamical fundamental fields one can of course get directly the Wilson loop in the fundamental by considering mesonic operators. Of course, one should also face the issue that the Wilson loop can be screened. If one is doing perturbation theory, or considering only the planar theory, then one does not need to worry about screening issues.

In this paper we assumed that the operators entering the correlator were such that the tree-level contribution to the correlator would always lead to a particle propagating from $x_{i}$ to $x_{i+1}$. One could easily imagine cases where this would not be the case. For example, one could have a charged chiral primary operator, such as $\operatorname{Tr}\left[\left(\phi_{1}+i \phi_{2}\right)^{2}\right]$ at $x_{i}$ and $x_{i+1}$. It is possible that one could slightly modify the discussion here so that these cases are also covered. In particular, one would have to understand whether we have the exchange of any particle between these two operators at higher loop level, etc.

In a parallel publication [50] an alternative way to take the light-cone limit is proposed. The correlators are first computed in four dimensions, and are then regularized by a "dual infrared" dimensional regulator. The limit produces the MHV gluon scattering amplitudes in the dual momentum space.

\section{Acknowledgments}

We would like to thank Nima Arkani-Hamed and Vladimir Braun for interesting discussions. GK is grateful to Vladimir Bazhanov and to the Australian National University, Canberra, and ES is grateful to Nima Arkani-Hamed and the Institute for Advanced Study, for warm hospitality at various stages of this work. This work was supported in part by the French Agence Nationale de la Recherche under grant ANR-06-BLAN-0142. This work was supported in part by U.S. Department of Energy grant \#DE-FG02-90ER40542.

\section{A Coordinate changes}

We have coordinates $Z^{M}=\left(Z^{-1}, Z^{0}, Z^{1}, Z^{2}, Z^{3}, Z^{4}\right)$, obeying $0=Z^{2}=-\left(Z^{-1}\right)^{2}-\left(Z^{0}\right)^{2}+$ $\left(Z^{1}\right)^{2}+\cdots+\left(Z^{4}\right)^{2}$. It is also convenient to introduce the following rotated coordinates

$$
\hat{Z}^{-1}=\frac{Z^{0}+Z^{-1}}{\sqrt{2}}, \quad \hat{Z}^{0}=\frac{Z^{0}-Z^{-1}}{\sqrt{2}}, \quad \hat{Z}^{i}=Z^{i}, \quad(i=1,2,3,4) .
$$


We then define the ordinary coordinates of $R^{1,3}$ as

$$
x^{\mu}=\frac{\hat{Z}^{\mu}}{G}, \quad G=\hat{Z}^{-1}+\hat{Z}^{4}, \quad(\mu=0,1,2,3) .
$$

We also introduce the $A d S_{3} \times S^{1}$ coordinates via

$$
\begin{aligned}
\tilde{G} & \equiv \sqrt{\left(Z^{3}\right)^{2}+\left(Z^{4}\right)^{2}}, & e^{i \varphi} & =\frac{Z^{3}+i Z^{4}}{\tilde{G}}, \\
\frac{Z^{0} \pm Z^{1}}{\tilde{G}} & =\cos \alpha e^{ \pm \gamma}, & \frac{Z^{-1} \pm Z^{2}}{\tilde{G}} & =\sin \alpha e^{ \pm \chi}
\end{aligned}
$$

We can think of the functions $G$ or $\tilde{G}$ as specifying a slice through the projective lightcone $Z^{2}=0$. The metric can then be written as $d s^{2}=d Z \cdot d Z=G^{2} d s^{2}$ where $d s^{2}$ is the metric in $A d S_{3} \times S^{1}$ or the metric of $R^{1,3}$ depending on whether we pick $G$ or $\tilde{G}$ in this formula.

These relations map the square polygon whose vertices are at $x^{\mu}=\left(1, \pm \frac{1}{\sqrt{2}}, 0,0\right)$, $\left(-1,0, \pm \frac{1}{\sqrt{2}}, 0\right)$ to a polygon whose null lines are at infinity in $\gamma$ and $\chi$. The propagator in any coordinate system is given by

$$
\langle\phi(1) \phi(2)\rangle=-\frac{G(1) G(2)}{2 Z_{1} \cdot Z_{2}}, \quad\langle\phi(1) \phi(2)\rangle_{\text {flat }}=\frac{1}{x_{12}^{2}}=-\frac{G(1) G(2)}{2 Z_{1} \cdot Z_{2}} .
$$

This formula can also be used to express distances in $R^{1,3}$ in terms of the $A d S_{3} \times S^{1}$ coordinates

$$
\begin{aligned}
x_{12}^{2} & =-\frac{2 Z_{1} \cdot Z_{2}}{G(1) G(2)}=-2 \frac{Z_{1} \cdot Z_{2}}{\tilde{G}(1) \tilde{G}(2)}\left(\frac{\tilde{G}(1) \tilde{G}(2)}{G(1) G(2)}\right)= \\
& =2\left[\sin \alpha_{1} \sin \alpha_{2} \cosh \chi_{12}+\cos \alpha_{1} \cos \alpha_{2} \cosh \gamma_{12}-1-\cos \varphi_{12}\right] \Omega(1) \Omega(2)
\end{aligned}
$$

with

$$
\Omega=\frac{\tilde{G}}{G}=\frac{1}{\frac{1}{\sqrt{2}}(\sin \alpha \cosh \chi+\cos \alpha \cosh \gamma)+\sin \varphi}
$$

We can also introduce $\gamma=\tau+\sigma, \chi=\tau-\sigma$ and $\alpha=\pi / 4+\beta$. Now the distance between two points at the same values of $\sigma, \sigma_{1}=\sigma_{2}=\sigma_{0} \gg 0$, at $\alpha_{1}=\alpha_{2}=\pi / 4$ and two different but large values of $\tau$ is

$$
x_{12}^{2} \sim 2 \frac{e^{\left|\tau_{12}\right|}}{e^{\left|\tau_{1}\right|+\sigma_{0}} e^{\left|\tau_{2}\right|+\sigma_{0}}} .
$$

If $\tau_{1,2}= \pm \tau_{0}$ then we see that $x_{12}^{2} \propto e^{-2 \sigma_{0}}$.

If we have four arbitrary and space-like separated points $x_{i},(i=1,2,3,4)$ we can map them via a conformal transformation to a rectangle in the $\tau$ and $\sigma$ plane at $\beta=\varphi=0$. The rectangle is characterize by its width and height, $\Delta \tau$ and $\delta \sigma$. We can then compute cross ratios by writing the coordinates $Z$ in terms of $\tau, \sigma$ as in (A.3) and then computing $\chi_{1234}=\frac{Z_{1} \cdot Z_{2} Z_{3} \cdot Z_{4}}{Z_{1} \cdot Z_{3} Z_{2} \cdot Z_{4}}$, etc. Using this we get to (4.8). 


\section{B Calculating the integral $\boldsymbol{I}_{\epsilon}(\boldsymbol{x})$}

Here we evaluate the function $I_{\epsilon}$ which appears in (3.21). To simplify the notation, we use translation invariance to set the external point $x_{0}=0$ and relabel the other points as follows:

$$
T^{\mu \nu}\left(x_{1}, x_{2}\right)=\partial_{1}^{[\mu} \partial_{2}^{\nu]} \int \frac{d^{4-2 \epsilon} x_{3}}{\left(-x_{13}^{2} x_{23}^{2} x_{3}^{2}\right)^{1-\epsilon}} .
$$

Introducing Feynman parameters, we find

$$
\begin{aligned}
T^{\mu \nu}\left(x_{1}, x_{2}\right) & =\partial_{1}^{[\mu} \partial_{2}^{\nu]} \prod_{i=1}^{3} \int_{0}^{\infty} \frac{(-i)^{1-\epsilon} d s_{i} s_{i}^{-\epsilon}}{\Gamma(1-\epsilon)} \int d^{4-2 \epsilon} x_{3} \mathrm{e}^{-i s_{1} x_{13}^{2}-i s_{2} x_{23}^{2}-i s_{3} x_{3}^{2}} \\
& =\frac{4 i^{2 \epsilon} \pi^{2-\epsilon} x_{1}^{[\mu} x_{2}^{\nu]}}{\Gamma^{3}(1-\epsilon)} \int_{0}^{\infty} \frac{d s_{1} d s_{2} d s_{3}\left(s_{1} s_{2} s_{3}\right)^{1-\epsilon}}{\left(s_{1}+s_{2}+s_{3}\right)^{3-\epsilon}} \exp \left(-i \frac{x_{12}^{2} s_{1} s_{2}+x_{1}^{2} s_{1} s_{3}+x_{2}^{2} s_{2} s_{3}}{s_{1}+s_{2}+s_{3}}\right) .
\end{aligned}
$$

Comparing to (3.21) and changing the integration variables $s_{i}=\lambda \beta_{i}$ (with $\lambda>0$ and $\sum_{i} \beta_{i}=1$ ), and after the elementary integration over $\lambda$ we obtain the following integral representation of the function $I_{\epsilon}$ :

$$
I_{\epsilon}\left(x_{1}, x_{2}\right)=-4 i \pi^{2-\epsilon} \frac{\Gamma(3-2 \epsilon)}{\Gamma^{3}(1-\epsilon)} \int_{0}^{1} \frac{[d \beta]_{3}\left(\beta_{1} \beta_{2} \beta_{3}\right)^{1-\epsilon}}{\left(-x_{12}^{2} \beta_{1} \beta_{2}+x_{1}^{2} \beta_{1} \beta_{3}+x_{2}^{2} \beta_{2} \beta_{3}\right)^{3-2 \epsilon}},
$$

with $\left[d \beta_{3}\right]=d \beta_{1} d \beta_{2} d \beta_{3} \delta\left(1-\beta_{1}-\beta_{2}-\beta_{3}\right)$. Changing once again the variables according to

$$
\beta_{1}=\frac{s}{1+z}, \quad \beta_{2}=\frac{1-s}{1+z}, \quad \beta_{3}=\frac{z}{1+z}
$$

with $0 \leq s \leq 1$ and $0 \leq z<\infty$, we obtain

$$
I_{\epsilon}\left(x_{1}, x_{2}\right)=-4 i \pi^{2-\epsilon} \frac{\Gamma(3-2 \epsilon)}{\Gamma^{3}(1-\epsilon)} \int_{0}^{\infty} \frac{d z z^{1-\epsilon}}{(1+z)^{3-\epsilon}} \int_{0}^{1} \frac{d s(s \bar{s})^{1-\epsilon}}{\left[-x_{12}^{2} s \bar{s}-z\left(x_{1}^{2} s+x_{2}^{2} \bar{s}\right)\right]^{3-2 \epsilon}}
$$

with $\bar{s}=1-s$.

We are interested in the leading asymptotic behavior of (B.4) for $x_{12}^{2} \rightarrow 0$. In this limit, replacing $z \rightarrow z x_{12}^{2} s \bar{s} /\left(x_{1}^{2} s+x_{2}^{2} \bar{s}\right)$ and noticing that $x_{1}^{2} s+x_{2}^{2} \bar{s}=\left(x_{1} s+x_{2} \bar{s}\right)^{2}$ for $x_{12}^{2}=0$, we find

$$
I_{\epsilon}\left(x_{1}, x_{2}\right) \rightarrow-4 i \pi^{2-\epsilon} \frac{\Gamma(2-\epsilon)}{\Gamma^{2}(1-\epsilon)}\left(-x_{12}^{2}\right)^{-1+\epsilon} \int_{0}^{1} d s\left[-\left(x_{1} s+x_{2} \bar{s}\right)\right]^{-2+\epsilon} \quad \text { for } x_{12}^{2} \rightarrow 0
$$

as announced in (3.23). Integrating with respect to $s$ yields

$$
I_{\epsilon}\left(x_{1}, x_{2}\right) \sim-\frac{4 i \pi^{2-\epsilon}}{\Gamma(1-\epsilon)}\left(-x_{12}^{2}\right)^{-1+\epsilon} \frac{\left(-x_{1}^{2}\right)^{-1+\epsilon}-\left(-x_{2}^{2}\right)^{-1+\epsilon}}{x_{1}^{2}-x_{2}^{2}} .
$$

For $\epsilon \rightarrow 0$ we recover (3.22). 


\section{Scalar propagator on the light cone}

In this appendix, we work out the first few terms in the light-cone expansion of the scalar propagator in an external gauge field, eqs. (2.7) and (2.10). We recall the basic assumption that the light-cone limit is taken so that $x^{2} \mu^{2} \ll 1$. This allows us to neglect the presence of the dimensional regularization cutoff $\mu^{2}$ in all formulas.

We start with the definition (2.6) and employ the proper time technique to write the solution to $(2.6)$ as (for $y=0)$

$$
S(x, 0 ; A)=\left\langle x\left|\frac{1}{i D^{2}}\right| 0\right\rangle=\int_{0}^{\infty} d s\left\langle x\left|\mathrm{e}^{-i s D^{2}}\right| 0\right\rangle .
$$

Here $D^{2}=D_{\mu}^{2}$ and $\left[D_{\mu}\right]^{a b}=\partial_{\mu} \delta^{a b}+g f^{a b c} A_{\mu}^{c}$ is the gauge covariant derivative in the adjoint representation. The expansion of $\left\langle x\left|\mathrm{e}^{-i s D^{2}}\right| 0\right\rangle$ in powers of the gauge field strength $F_{\mu \nu}$ looks as [51]

$$
\begin{aligned}
\left\langle x\left|\mathrm{e}^{-i s D^{2}}\right| 0\right\rangle= & \left\langle x\left|\mathrm{e}^{-i s \partial^{2}}\right| 0\right\rangle\left\{[x, 0]+s \int_{0}^{1} d u u \bar{u}[x, u x] x_{\nu} D_{\mu} F^{\mu \nu}(u x)[u x, 0]\right. \\
& \left.+2 i s \int_{0}^{1} d u \bar{u} \int_{0}^{1} d v v x_{\lambda} x_{\rho}[x, u x] F^{\lambda \xi}(u x)[u x, v x] F_{\xi}^{\rho}(v x)[v x, 0]+O\left(s^{2}\right)\right\}
\end{aligned}
$$

where $[x, y] \equiv P \exp \left(i \int_{y}^{x} d z \cdot A(z)\right)$ stands for a Wilson line in the adjoint representation connecting points $x$ and $y$, and $\bar{u}=1-u$.

The first factor on the right-hand side of (C.2) can be easily evaluated by going to the momentum representation

$$
\left\langle x\left|\mathrm{e}^{-i s \partial^{2}}\right| 0\right\rangle=\int \frac{d^{4} p}{(2 \pi)^{4}} \mathrm{e}^{-i p x} \mathrm{e}^{i s p^{2}} \sim s^{-2} \mathrm{e}^{-i x^{2} /(4 s)} .
$$

Its substitution into (C.1) yields the free propagator

$$
S^{\operatorname{tree}}(x)=\int_{0}^{\infty} d s\left\langle x\left|\mathrm{e}^{-i s \partial^{2}}\right| 0\right\rangle=-\frac{1}{(2 \pi)^{2} x^{2}} .
$$

Likewise, substituting (C.2) into (C.1) and performing the $s$-integration term by term, we find that the expansion of (C.2) in $s$ can be translated into a similar expansion of $S(x, 0 ; A)$ in $x^{2}$ :

$$
S(x, 0 ; A)=-\frac{1}{(2 \pi)^{2} x^{2}} P \exp \left(i g \int_{0}^{x} d z \cdot A(z)\right)\left[1+x^{2}\left(\mathcal{O}_{1}(x)+\mathcal{O}_{2}(x)\right)+O\left(x^{4}\right)\right],
$$

where $\mathcal{O}_{1}$ and $\mathcal{O}_{2}$ are the so-called light-ray operators $[51,52]$

$$
\begin{aligned}
\mathcal{O}_{1} & \sim \int_{0}^{1} d u u \bar{u}[0, u x] x_{\nu} D_{\mu} F^{\mu \nu}(u x)[u x, 0] \\
\mathcal{O}_{2} & \sim 2 i \int_{0}^{1} d u \bar{u} \int_{0}^{1} d v v x_{\lambda} x_{\rho}[0, u x] F^{\lambda \xi}(u x)[u x, v x] F^{\rho}{ }_{\xi}(v x)[v x, 0] .
\end{aligned}
$$


These operators can be thought of as generating functions for twist-two operators, e.g.

$$
\mathcal{O}_{1} \sim \int_{0}^{1} d u u \bar{u} \mathrm{e}^{u(x \cdot D)} x_{\nu} D_{\mu} F^{\mu \nu}(0)=\sum_{N \geq 0} \frac{N+1}{(N+3) !}(x \cdot D)^{N} x_{\nu} D_{\mu} F^{\mu \nu}(0) .
$$

We conclude from (C.5) that the leading contribution to the scalar propagator for $x^{2} \rightarrow 0$ comes from the first term in the square brackets corresponding to the identity operator with twist zero.

\section{A factor of two}

In this appendix we explain the relative factor of two which arises in front of double logarithmic terms when we compute the Wilson loop in dimensional regularization versus the case when we compute the limit of the correlation functions and set the distances equal to $x_{i, i+1}^{2}=\mu^{-2}$. For the most divergent terms we get

$$
\begin{aligned}
\left.\log \langle O(1) \cdots O(n)\rangle\right|_{x_{i, i+1}^{2}=\mu^{-2}} & \sim-n \frac{\Gamma_{\text {cusp }}}{2}\left(\log \mu^{2}\right)^{2}, \\
\log W_{\text {dim-reg }}^{\text {adj }} & \sim-n \frac{\Gamma_{\text {cusp }}}{4}\left(\log \mu^{2}\right)^{2},
\end{aligned}
$$

where $n$ is the number of cusps. Naively one would have expected that this most divergent term should have matched.

The relative factor of two has a simple geometric explanation if we go to the coordinates we introduced in appendix A. For simplicity consider the case of the square, which contains four cusps.

Let us set the operators in the correlation function at the vertices of a square at $\tau= \pm \sigma= \pm \sigma_{0}$. Then one finds that the distances are

$$
x_{12}^{2} \approx e^{-2 \sigma_{0}} \sim \mu^{-2}, \quad x_{23}^{2} \approx e^{-2 \tau_{0}} \sim \mu^{-2}, \quad \tau_{0}=\sigma_{0}
$$

The total area of the rectangle is $4 \sigma_{0}^{2}$. This agrees with (D.1), for $n=4$, once we use (D.3).

We now consider a Wilson loop in the dimensionally regularized theory whose cusps are light-like separated. Under the conformal mapping the edges of the null square polygon are sent to $\tau= \pm \infty$ and $\sigma= \pm \infty$. The coupling constant becomes $\tau$ and $\sigma$ dependent because the theory is not confomally invariant. One can do conformal transformations in a non-conformal theory if one remembers to change the coupling constants that violate the conformal symmetry. In order to find the new coupling constant we can do the following. The coupling in the dimensionally regularized theory has a scale $\mu$. For our purposes, we can replace $\mu \rightarrow \mu \Omega$, where $\Omega$ is given in (A.6) and is equal to $\Omega \sim e^{-|\tau|-|\sigma|}$ for large $\tau$ and $\sigma$. Thus we end up with a coupling that depends on the position and it is such that it goes to zero for large $\tau, \sigma$. Thus, as expected, we get a finite answer for the contribution of the electric field, since the energy in the electric flux is proportional to the coupling. The coupling is turning itself off when $\mu \Omega \sim 1$. Thus we find that $|\tau|+|\sigma| \leq \log \mu=\sigma_{0}$. This gives the diagonal lines in figure 10 . These produces a square of size $2 \sigma_{0}^{2}$, in agreement with (D.2), with $n=4$. 


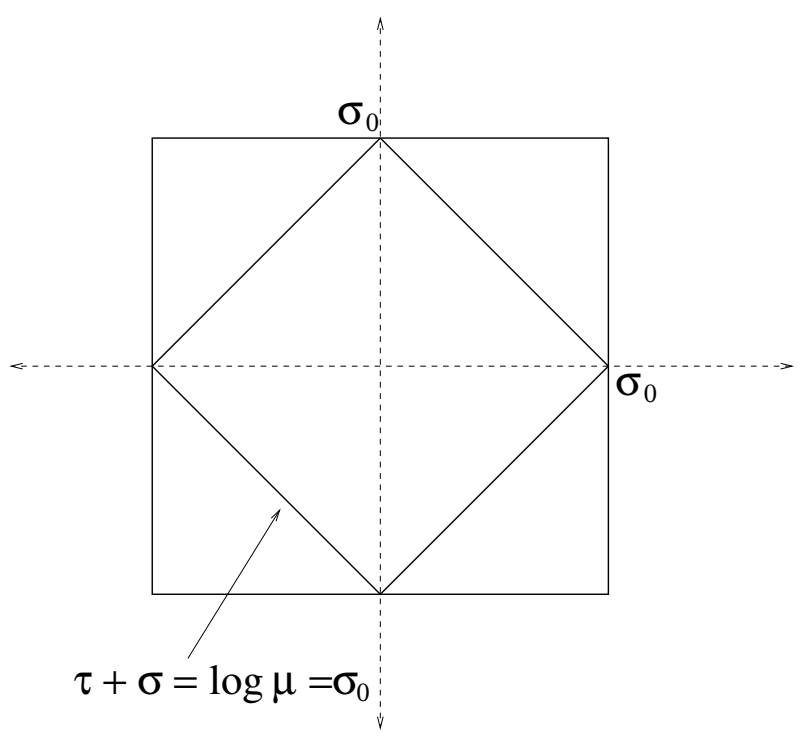

Figure 10. Origin of a factor of two due to the different ways of regularizing the contribution of the color electric flux. In the case of correlation function we have operators sitting at the vertices of the outer square. Fast propagating particles define the outersquare and the contribution of the flux is the area of this square. In dimensional regularization we end up with a coupling constant that depends on scale in these new coordinates. The coupling switches itself off outside the small square. Thus the contribution is just the area of the small square.

This explanation is completely general and is valid whenever the formulas (D.1) and (D.2) are valid. This same factor of two was observed in the closely related problem of QCD form factors in [53, 54].

Open Access. This article is distributed under the terms of the Creative Commons Attribution Noncommercial License which permits any noncommercial use, distribution, and reproduction in any medium, provided the original author(s) and source are credited.

\section{References}

[1] A.M. Polyakov, Gauge fields as rings of glue, Nucl. Phys. B 164 (1980) 171.

[2] G.P. Korchemsky and A.V. Radyushkin, Renormalization of the Wilson Loops Beyond the Leading Order, Nucl. Phys. B 283 (1987) 342 [SPIRES].

[3] I.A. Korchemskaya and G.P. Korchemsky, On light-like Wilson loops, Phys. Lett. B 287 (1992) 169 [SPIRES].

[4] A. Bassetto, I.A. Korchemskaya, G.P. Korchemsky and G. Nardelli, Gauge invariance and anomalous dimensions of a light cone Wilson loop in lightlike axial gauge, Nucl. Phys. B 408 (1993) 62 [hep-ph/9303314] [SPIRES].

[5] A. Sen, Asymptotic Behavior of the Sudakov Form-Factor in QCD, Phys. Rev. D 24 (1981) 3281 [SPIRES].

[6] G.P. Korchemsky and A.V. Radyushkin, Loop Space Formalism And Renormalization Group For The Infrared Asymptotics Of QCD, Phys. Lett. B 171 (1986) 459 [SPIRES]. 
[7] S.V. Ivanov, G.P. Korchemsky and A.V. Radyushkin, Infrared Asymptotics Of Perturbative QCD: Contour Gauges, Yad. Fiz. 44 (1986) 230 [Sov. J. Nucl. Phys. 44 (1986) 145] [SPIRES].

[8] G.P. Korchemsky and A.V. Radyushkin, Infrared asymptotics of perturbative QCD. Quark and gluon propagators, Sov. J. Nucl. Phys. 45 (1987) 127 [Yad. Fiz. 45 (1987) 198] [SPIRES].

[9] G.P. Korchemsky and A.V. Radyushkin, Infrared asymptotics of perturbative QCD. Vertex functions, Sov. J. Nucl. Phys. 45 (1987) 910 [Yad. Fiz. 45 (1987) 1466] [SPIRES].

[10] L.F. Alday and J.M. Maldacena, Gluon scattering amplitudes at strong coupling, JHEP 06 (2007) 064 [arXiv: 0705.0303] [SPIRES].

[11] G.P. Korchemsky, J.M. Drummond and E. Sokatchev, Conformal properties of four-gluon planar amplitudes and Wilson loops, Nucl. Phys. B 795 (2008) 385 [arXiv:0707.0243] [SPIRES].

[12] A. Brandhuber, P. Heslop and G. Travaglini, MHV Amplitudes in $N=4$ Super Yang-Mills and Wilson Loops, Nucl. Phys. B 794 (2008) 231 [arXiv:0707.1153] [SPIRES].

[13] J.M. Drummond, J. Henn, G.P. Korchemsky and E. Sokatchev, On planar gluon amplitudes/Wilson loops duality, Nucl. Phys. B 795 (2008) 52 [arXiv:0709.2368] [SPIRES].

[14] L.F. Alday and J. Maldacena, Comments on gluon scattering amplitudes via AdS/CFT, JHEP 11 (2007) 068 [arXiv:0710.1060] [SPIRES].

[15] J.M. Drummond, J. Henn, G.P. Korchemsky and E. Sokatchev, Conformal Ward identities for Wilson loops and a test of the duality with gluon amplitudes,

Nucl. Phys. B 826 (2010) 337 [arXiv:0712.1223] [SPIRES].

[16] Z. Bern et al., The Two-Loop Six-Gluon MHV Amplitude in Maximally Supersymmetric Yang-Mills Theory, Phys. Rev. D 78 (2008) 045007 [arXiv:0803.1465] [SPIRES].

[17] J.M. Drummond, J. Henn, G.P. Korchemsky and E. Sokatchev, Hexagon Wilson loop = six-gluon MHV amplitude, Nucl. Phys. B 815 (2009) 142 [arXiv:0803.1466] [SPIRES].

[18] J.M. Drummond, J. Henn, G.P. Korchemsky and E. Sokatchev, Dual superconformal symmetry of scattering amplitudes in $N=4$ super-Yang-Mills theory,

Nucl. Phys. B 828 (2010) 317 [arXiv:0807.1095] [SPIRES].

[19] N. Berkovits and J. Maldacena, Fermionic T-duality, Dual Superconformal Symmetry and the Amplitude/Wilson Loop Connection, JHEP 09 (2008) 062 [arXiv:0807.3196] [SPIRES].

[20] N. Beisert, R. Ricci, A.A. Tseytlin and M. Wolf, Dual Superconformal Symmetry from AdS5 $x$ S5 Superstring Integrability, Phys. Rev. D 78 (2008) 126004 [arXiv:0807.3228] [SPIRES].

[21] D.J. Gross and S.B. Treiman, Light cone structure of current commutators in the gluon quark model, Phys. Rev. D 4 (1971) 1059 [SPIRES].

[22] L. Andrianopoli and S. Ferrara, $K-K$ excitations on $A d S_{5} \times S^{5}$ as $N=4{ }^{*}$ primary* superfields, Phys. Lett. B 430 (1998) 248 [hep-th/9803171] [SPIRES].

[23] J.M. Maldacena, The large- $N$ limit of superconformal field theories and supergravity, Int. J. Theor. Phys. 38 (1999) 1113 [Adv. Theor. Math. Phys. 2 (1998) 231] [hep-th/9711200] [SPIRES].

[24] S.S. Gubser, I.R. Klebanov and A.M. Polyakov, Gauge theory correlators from non-critical string theory, Phys. Lett. B 428 (1998) 105 [hep-th/9802109] [SPIRES]. 
[25] E. Witten, Anti-de Sitter space and holography, Adv. Theor. Math. Phys. 2 (1998) 253 [hep-th/9802150] [SPIRES].

[26] S. Penati, A. Santambrogio and D. Zanon, Two-point functions of chiral operators in $N=4$ SYM at order $g^{4}$, JHEP 12 (1999) 006 [hep-th/9910197] [SPIRES].

[27] S. Penati, A. Santambrogio and D. Zanon, More on correlators and contact terms in $N=4$ SYM at order $g^{4}$, Nucl. Phys. B 593 (2001) 651 [hep-th/0005223] [SPIRES].

[28] E. D'Hoker, D.Z. Freedman and W. Skiba, Field theory tests for correlators in the AdS/CFT correspondence, Phys. Rev. D 59 (1999) 045008 [hep-th/9807098] [SPIRES].

[29] S. Lee, S. Minwalla, M. Rangamani and N. Seiberg, Three-point functions of chiral operators in $D=4, N=4 S Y M$ at large- $N$, Adv. Theor. Math. Phys. 2 (1998) 697 [hep-th/9806074] [SPIRES].

[30] P.S. Howe, E. Sokatchev and P.C. West, 3-point functions in $N=4$ Yang-Mills, Phys. Lett. B 444 (1998) 341 [hep-th/9808162] [SPIRES].

[31] B. Eden, C. Schubert and E. Sokatchev, Three-loop four-point correlator in $N=4 S Y M$, Phys. Lett. B 482 (2000) 309 [hep-th/0003096] [SPIRES].

[32] B. Eden, C. Schubert and E. Sokatchev, unpublished (2000).

[33] M. Bianchi, S. Kovacs, G. Rossi and Y.S. Stanev, Anomalous dimensions in N=4 SYM theory at order $g^{4}$, Nucl. Phys. B 584 (2000) 216 [hep-th/0003203] [SPIRES].

[34] B. Eden, P.S. Howe, C. Schubert, E. Sokatchev and P.C. West, Four-point functions in $N=4$ supersymmetric Yang-Mills theory at two loops, Nucl. Phys. B 557 (1999) 355 [hep-th/9811172] [SPIRES].

[35] B. Eden, P.S. Howe, C. Schubert, E. Sokatchev and P.C. West, Simplifications of four-point functions in $N=4$ supersymmetric Yang-Mills theory at two loops, Phys. Lett. B 466 (1999) 20 [hep-th/9906051] [SPIRES].

[36] A. Gorsky and A. Zhiboedov, One-loop derivation of the Wilson polygon - MHV amplitude duality, J. Phys. A 42 (2009) 355214 [arXiv:0904.0381] [SPIRES].

[37] V.M. Braun, G.P. Korchemsky and D. Mueller, The uses of conformal symmetry in QCD, Prog. Part. Nucl. Phys. 51 (2003) 311 [hep-ph/0306057] [SPIRES].

[38] L.F. Alday and J.M. Maldacena, Comments on operators with large spin, JHEP 11 (2007) 019 [arXiv:0708.0672] [SPIRES].

[39] L.F. Alday, D. Gaiotto and J. Maldacena, Thermodynamic Bubble Ansatz, JHEP 09 (2011) 032 [arXiv:0911.4708] [SPIRES].

[40] F. Gonzalez-Rey, I.Y. Park and K. Schalm, A note on four-point functions of conformal operators in $N=4$ super-Yang-Mills, Phys. Lett. B 448 (1999) 37 [hep-th/9811155] [SPIRES].

[41] N. Drukker and J. Plefka, Superprotected n-point correlation functions of local operators in $N=4$ super Yang-Mills, JHEP 04 (2009) 052 [arXiv:0901.3653] [SPIRES].

[42] N. Drukker and J. Plefka, The structure of n-point functions of chiral primary operators in $N=4$ super Yang-Mills at one-loop, JHEP 04 (2009) 001 [arXiv:0812.3341] [SPIRES].

[43] N.I. Usyukina and A.I. Davydychev, An Approach to the evaluation of three and four point ladder diagrams, Phys. Lett. B 298 (1993) 363 [SPIRES]. 
[44] N.I. Usyukina and A.I. Davydychev, Exact results for three and four point ladder diagrams with an arbitrary number of rungs, Phys. Lett. B 305 (1993) 136 [SPIRES].

[45] Z. Bern, L.J. Dixon, D.C. Dunbar and D.A. Kosower, One-Loop n-Point Gauge Theory Amplitudes, Unitarity and Collinear Limits, Nucl. Phys. B 425 (1994) 217 [hep-ph/9403226] [SPIRES].

[46] F.A. Dolan and H. Osborn, Conformal partial wave expansions for $N=4$ chiral four point functions, Annals Phys. 321 (2006) 581 [hep-th/0412335] [SPIRES].

[47] G.P. Korchemsky, Asymptotics of the Altarelli-Parisi-Lipatov Evolution Kernels of Parton Distributions, Mod. Phys. Lett. A 4 (1989) 1257 [SPIRES].

[48] G.P. Korchemsky and G. Marchesini, Structure function for large $x$ and renormalization of Wilson loop, Nucl. Phys. B 406 (1993) 225 [hep-ph/9210281] [SPIRES].

[49] L.F. Alday, D. Gaiotto, J. Maldacena, A. Sever and P. Vieira, An Operator Product Expansion for Polygonal null Wilson Loops, JHEP 04 (2011) 088 [arXiv:1006.2788] [SPIRES].

[50] B. Eden, G.P. Korchemsky and E. Sokatchev, From correlation functions to scattering amplitudes, arXiv:1007.3246 [SPIRES].

[51] I.I. Balitsky and V.M. Braun, Evolution Equations for QCD String Operators, Nucl. Phys. B 311 (1989) 541 [SPIRES].

[52] D. Mueller, D. Robaschik, B. Geyer, F.M. Dittes and J. Horejsi, Wave functions, evolution equations and evolution kernels from light-ray operators of QCD, Fortschr. Phys. 42 (1994) 101 [hep-ph/9812448] [SPIRES].

[53] G.P. Korchemsky, Sudakov Form-Factor In QCD, Phys. Lett. B 220 (1989) 629 [SPIRES].

[54] G.P. Korchemsky, Double Logarithmic Asymptotics in QCD, Phys. Lett. B 217 (1989) 330 [SPIRES]. 